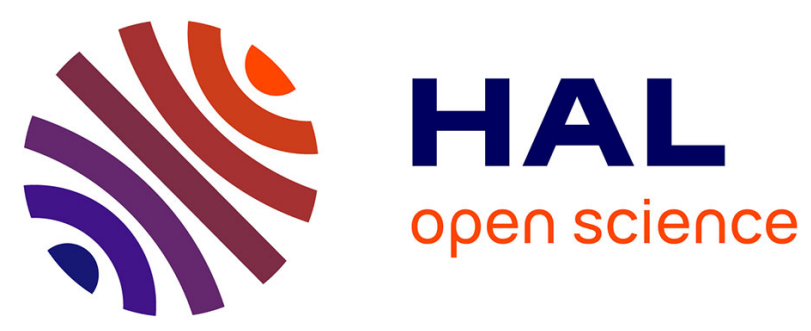

\title{
Calculations of low-frequency radio emission by cosmic ray-induced particle showers
}

Daniel García-Fernández, Benoît Revenu, Didier Charrier, Richard Dallier, Antony Escudie, Lilian Martin

\section{- To cite this version:}

Daniel García-Fernández, Benoît Revenu, Didier Charrier, Richard Dallier, Antony Escudie, et al.. Calculations of low-frequency radio emission by cosmic ray-induced particle showers. Phys.Rev.D, 2018, 97 (10), pp.103010. 10.1103/PhysRevD.97.103010 . hal-01802100

\section{HAL Id: hal-01802100 \\ https://hal.science/hal-01802100}

Submitted on 15 Jun 2020

HAL is a multi-disciplinary open access archive for the deposit and dissemination of scientific research documents, whether they are published or not. The documents may come from teaching and research institutions in France or abroad, or from public or private research centers.
L'archive ouverte pluridisciplinaire HAL, est destinée au dépôt et à la diffusion de documents scientifiques de niveau recherche, publiés ou non, émanant des établissements d'enseignement et de recherche français ou étrangers, des laboratoires publics ou privés. 


\title{
Calculations of low-frequency radio emission by cosmic-ray-induced particle showers
}

\author{
Daniel García-Fernández, ${ }^{1}$ Benoît Revenu, ${ }^{1,2}$ Didier Charrier, ${ }^{1,2}$ Richard Dallier, ${ }^{1,2}$ Antony Escudie, ${ }^{1}$ and Lilian Martin ${ }^{1,2}$ \\ ${ }^{1}$ Subatech, Institut Mines-Télécom Atlantique, CNRS, Université de Nantes, Nantes 44300, France \\ ${ }^{2}$ Unité Scientifique de Nançay, Observatoire de Paris, CNRS, PSL, UO/OSUC, Nançay 18330, France
}

(Received 2 November 2017; published 16 May 2018)

\begin{abstract}
The radio technique for the detection of high-energy cosmic rays consists in measuring the electric field created by the particle showers created inside a medium by the primary cosmic ray. The electric field is then used to infer the properties of the primary particle. Nowadays, the radio technique is a standard, wellestablished technique. While most current experiments measure the field at frequencies above $20 \mathrm{MHz}$, several experiments have reported a large emission at low frequencies, below $10 \mathrm{MHz}$. The EXTASIS experiment aims at measuring again and understanding this low-frequency electric field. Since at low frequencies the standard far-field approximation for the calculation of the electric field does not necessarily hold, in order to comprehend the low-frequency emission we need to go beyond the far-field approximation. We present in this work a formula for the electric field created by a particle track inside a dielectric medium that is valid for all frequencies. We then implement this formula in the SELFAS Monte Carlo code and calculate the low-frequency electric field of the extensive air shower (EAS). We also study the electric field of a special case of the transition radiation mechanism when the EAS particles cross the air-soil boundary. We introduce the sudden death pulse, the direct emission caused by the coherent deceleration of the shower front at the boundary, as a first approximation to the whole electric field for the air-soil transition, and study its properties. We show that at frequencies larger than $20 \mathrm{MHz}$ and distances larger than $100 \mathrm{~m}$, the standard far-field approximation for the horizontal polarizations of the field is always accurate at the $1 \%$ level.
\end{abstract}

DOI: $10.1103 /$ PhysRevD.97.103010

\section{INTRODUCTION}

Cosmic rays with energies $>10^{15} \mathrm{eV}$ cannot be directly measured due to their low flux, so they are detected via the particle showers they create when they enter the atmosphere. The secondary particles present in the extensive air shower (EAS) can give us information on the primary cosmic ray, such as its arrival direction, its energy and, more recently, its composition. There are three main detection techniques for inspecting the EAS and elucidating the properties of the primary particle. First, there is the surface detection technique, wherein the shower particles arriving at ground level can be detected, and this sample of the shower is then used to analyze the properties of the primary. Second, there is the fluorescence technique. Whenever a particle shower passes through the atmosphere, the nitrogen molecules within it become excited and emit fluorescence light when they deexcite. This fluorescent light can be detected on moonless nights, and its luminosity is proportional to the number of charged particles traversing a given region of the atmosphere, allowing us to reconstruct the development of the EAS. And third, we have the radio technique.

The radio detection technique of high-energy particles consists in detecting, by the use of radio antennas, the electric field created by the charged particles present in a particle shower. The amplitude, ground footprint, arrival time, and polarization of the electric field can be correlated to the arrival direction, energy, and even composition of the primary particle [1-3]. The radio technique is a wellestablished technique as of today [4]. This technique was proposed in 1962 by G. Askaryan as a method for detecting cosmic rays and neutrinos [5]. It did not take long to observe pulses correlated with showers measured by air shower arrays [6], but it was abandoned due to several limitations that prevented it from being as competitive as the surface detection. With the advent of modern electronics, radio detection and the interest it generated were reawakened.

We know today that the electric field created by EAS can be explained by the superposition of two effects. The dominant effect is called the geomagnetic effect and it is induced by the deflection of the charged particles by the geomagnetic field. Positive and negative charges deviate in opposite directions, creating a total current in the same direction that produces an electromagnetic field with a polarization along the Lorentz force direction, $\mathbf{v} \times \mathbf{B}$, with $\mathbf{v}$ being the shower axis and $\mathbf{B}$ the geomagnetic field. The Askaryan effect is related to the appearance of an excess of negative charge (electrons) within the shower, which creates detectable electric field with a polarization radial with respect to the shower axis. We can find in [7] a theoretical description of these two effects and in [8-10] data that evidence their existence and interplay to 
create the total electric field. Since a significant part of the shower particles travel faster than the speed of light, relativistic interference effects are also relevant, leading to the appearance of a Cherenkov cone.

Most radio experiments detecting air showers nowadays (for instance, CODALEMA [11], AERA [12], Tunka-Rex [13] or LOFAR [14]) measure the electric field in the [20-80] MHz band. This is due to the fact that the field from EAS is usually coherent up to several tens of $\mathrm{MHz}$, and above $80 \mathrm{MHz}$ we find FM radio emitters. However, near the Cherenkov angle, the field can be coherent up to $\mathrm{GHz}$ frequencies [15-17]. There are some complications for measuring below $20 \mathrm{MHz}$, mainly the atmospheric and galactic noise, and that is why these experiments have chosen the aforementioned band.

Several experiments in the past have informed us of the observation of pulses correlated with air showers at frequencies below $20 \mathrm{MHz}$, but the results are not well understood. The first detection of this kind took place as early as in 1967 [18]. In 1970, research at Haverah Park found an average low-frequency signal (at $3.6 \mathrm{MHz}$ ) of $300 \mu \mathrm{V} \mathrm{m}^{-1} \mathrm{MHz}^{-1}$, averaged over 400 showers [19]. However, a repeat run gave a null result [20]. In 1971 [20], a signal of about $1 \mu \mathrm{V} \mathrm{m}^{-1} \mathrm{MHz}^{-1}$ at $1.98 \mathrm{MHz}$ was measured. According to the author's estimation, the shower energy was $\sim 2 \times 10^{14} \mathrm{eV}$, which is incredibly low for a radio-detected shower. This energy estimation allows the author to conclude that a $10^{17} \mathrm{eV}$ shower should present a field strength of $500 \mu \mathrm{V} \mathrm{m}^{-1} \mathrm{MHz}^{-1}$, but the detection of a $\sim 2 \times 10^{14} \mathrm{eV}$ shower by a radio array seems implausible even by today's standards. In [21], a mean field of $2.5 \pm$ $0.6 \mu \mathrm{V} \mathrm{m}^{-1} \mathrm{MHz}^{-1}$ was measured at $3.6 \mathrm{MHz}$, and with the help of the shower size estimation and the hypothesis of pure geomagnetic emission, the authors extrapolate $300 \mu \mathrm{V} \mathrm{m}^{-1} \mathrm{MHz}^{-1}$ at $10^{17}$, more than 1 order of magnitude larger than the standard $20-80 \mathrm{MHz}$ emission. We must point out that the measured fields so far had been of the order of a few $\mu \mathrm{V} \mathrm{m}^{-1} \mathrm{MHz}^{-1}$. In fact, [22] reports an upper limit of $0.6 \mu \mathrm{V} \mathrm{m}^{-1} \mathrm{MHz}^{-1}$ for a $10^{17} \mathrm{eV}$ shower, obtained with an antenna located $\lambda / 10$ wavelengths above ground, which is incompatible with previous estimations.

In spite of the controversy, later experiments reported direct measurements of large electric fields. The Akeno experiment [23] pursued even lower frequencies (26$300 \mathrm{kHz}$ ) and found correlation of signals in that band with signals at higher frequencies, with a typical amplitude of hundreds of $\mu \mathrm{V} \mathrm{m}^{-1} \mathrm{MHz}^{-1}$, a measure the authors repeated in [24]. EAS-RADIO [25], set up at EASTOP, was able to measure again fields of the same order.

A discussion of these and more experiments and a brief theoretical summary can be found in [26]. The experiments and analyses cited therein agree that the standard calculations used in radio experiments are not enough to describe the measured field. Some mechanisms, such as transition radiation created by an infinite track or deviation of particles in the geoelectric field, do not seem to be favored by the data $[23,27]$. Coherent deceleration of the shower front, also called sudden death pulse (SDP) has been proposed in $[23,26,28]$ as a means to explain the low-frequency emission.

This SDP is related to the transition radiation (TR) field. A transition radiation field is, in general, the electric radiation field created by a charge that travels within several media. Since it is a radiation field, by definition, it implies the far-field regime. In our case, we want to calculate also the near-field component, so our problem is rather the calculation of a transition field that reduces to TR in the far field. Most closed formulas for TR, for instance, Ginzburg's formula, are valid for particles traveling on a straight infinite line. These formulas can be applied as long as the particle trajectory begins and ends at a distance from the interface much greater than the observation wavelength. Particles in an EAS are stopped in a few $\mathrm{cm}$ of soil, and observation wavelengths for ground-based radio experiments are always much larger than $30 \mathrm{~cm}$, so we cannot rely on these analytical formulas for our case even if the observer is far away from the shower core. As an approximation, we assume that the shower stops immediately when it reaches the air-soil boundary and we calculate only the direct field without the effect of the boundary. We call SDP approximation the field calculated with these assumptions. When the observer is far enough from the shower core, the standard shower emission is separated from the SDP and we can identify it by its delay.

In order to measure again the electric field of an EAS at low frequencies and shed light on the emission mechanisms, the EXTASIS experiment has been set up at the Nançay radio observatory. With a frequency band spanning from 1.7 to $3.7 \mathrm{MHz}$ and seven antennas with vertical and horizontal polarizations hung at the top of $9 \mathrm{~m}$ high masts, EXTASIS has already detected low-frequency pulses correlated with pulses in the [20-200] $\mathrm{MHz}$ band and with events seen by a surface detector [29]. The data look like a real cosmic-ray event, and the reconstruction of the arrival direction in the [1.7-3.7] $\mathrm{MHz}$ band is in agreement with the reconstruction in the [20-200] MHz band and the one given by the surface detector. These data also hint at a larger reach of the electric field at low frequencies. EXTASIS has also among its goals the detection of the transition field created when the shower reaches the ground.

One of the problems that arises is the correct calculation of the electric field. Most of the theoretical formulas $[30,31]$ and standard codes used, such as SELFAS [32], ZHAireS [33], or CoREAS [34], use the far-field approximation to obtain the radiation field only. The far-field approximation holds when the wave number $k$ and the distance from emitter to observer verifies $k R=2 \pi R / \lambda \gg 1$, which allows us to approximate the electric field as a pure radiation field. At $\sim 1 \mathrm{MHz}$, the wavelength is $\sim 300 \mathrm{~m}$, which is comparable to the distance from the measuring antenna to the shower core and axis. If, moreover, we expect a noticeable emission from the shower close to the core (as in the sudden death mechanism), 
the far-field approximation could not suffice. In particular, for an antenna at $100 \mathrm{~m}$ from the shower core, $k R=$ $2 \pi \cdot 100 / 300 \sim 2$, so near-field effects could be important at the EXTASIS frequencies.

We begin by deriving in Sec. II a time-domain formula for the field of a particle track valid for all frequencies. We derive again the formula in frequency domain in Sec. III. The time-domain and frequency-domain formulas are compared to the far-field approximation or Zas-HalzenStanev (ZHS) formula [30,35]. In Sec. IV we explain the implementation of the exact formula in the SELFAS Monte Carlo (MC) code. In Sec. V we show some examples of the predictions of this formula for the emission coming from EAS and give a range of validity for the farfield approximation. We introduce the coherent deceleration of the shower front or sudden death pulse and study its properties in Sec. VI.

\section{ELECTRIC FIELD OF A PARTICLE TRACK IN TIME DOMAIN}

\section{A. Derivation of the formula in time domain}

Our aim is to derive a formula for the electric field emitted by a particle contained inside a shower. Since we are operating at the quantum scale, we must justify the use of classical electrodynamics. The trajectory of the particle inside a particle shower is created by the interactions with the medium that cause the particle to change direction and speed almost instantaneously. Physically, however, there must be a time related to the interaction below which the use of quantum theory is imperative. Let us call this time $\Delta t_{q}$. We expect the frequency components of the field to be valid until the observation frequency is comparable to $1 / \Delta t_{q}$, and in this low-frequency region we can apply classical electrodynamics [36]. Let us assume $\Delta t_{q}$ is related to the Compton wavelength $\lambda_{C}$ of the electron,

$\Delta t_{q} \sim \frac{h}{m_{e} c^{2}}=\frac{\lambda_{C}}{c}=\frac{2.425 \times 10^{-12} \mathrm{~m}}{0.3 \mathrm{~m} \mathrm{~ns}^{-1}}=8.09 \times 10^{-12} \mathrm{~ns}$,

which implies that the order of magnitude of the maximum frequency where the classical approach should work is

$$
\nu_{\text {classical }} \ll \frac{1}{\Delta t_{q}} \sim \frac{m_{e} c^{2}}{h}=\frac{c}{\lambda_{C}} \sim 1.24 \times 10^{11} \mathrm{GHz} .
$$

We are interested in radio emission below the $\mathrm{THz}$, so the use of classical electrodynamics is justified.

We begin with Maxwell's equations for the potentials inside a homogeneous, dielectric medium with permittivity $\epsilon$, independent of the frequency. Its refractive index is, therefore, $n=\sqrt{\epsilon / \epsilon_{0}}$, and the speed of light in the medium is $c_{n}=c / n$. Using the Lorenz gauge,

$$
\nabla^{2} \Phi-\frac{1}{c_{n}^{2}} \frac{\partial^{2} \phi}{\partial t^{2}}=-\frac{\rho}{\epsilon},
$$

$$
\nabla^{2} \mathbf{A}-\frac{1}{c_{n}^{2}} \frac{\partial^{2} \mathbf{A}}{\partial t^{2}}=-\mu_{0} \mathbf{J}
$$

where $t$ is the observer time, $\Phi$ and $\mathbf{A}$ are the electromagnetic potentials, $\rho$ is the charge density, and $\mathbf{J}$ is the current density. Equations (3) and (4) are similar to their vacuum counterparts. Formally, we have made the changes $\epsilon_{0} \rightarrow \epsilon$ and $c \rightarrow c_{n}$, so the formal solutions for the potential are the usual retarded potentials with these substitutions,

$$
\begin{gathered}
\Phi(\mathbf{x}, t)=\frac{1}{4 \pi \epsilon} \int \mathrm{d}^{3} x^{\prime} \frac{1}{R}\left[\rho\left(\mathbf{x}^{\prime}, t^{\prime}\right)\right]_{\mathrm{ret}}, \\
\mathbf{A}(\mathbf{x}, t)=\frac{\mu_{0}}{4 \pi} \int \mathrm{d}^{3} x^{\prime} \frac{1}{R}\left[\mathbf{J}\left(\mathbf{x}^{\prime}, t^{\prime}\right)\right]_{\mathrm{ret}},
\end{gathered}
$$

where $R=\left|\mathbf{x}-\mathbf{x}^{\prime}\right|$ and $t^{\prime}$ is the retarded or emission time, defined implicitly as

$$
t^{\prime}+\frac{1}{c_{n}}\left|\mathbf{x}-\mathbf{x}^{\prime}\left(t^{\prime}\right)\right|=t .
$$

As long as our source is not superluminous, that is, as long as its speed is less than $c_{n}$, the speed of light in the medium, the calculation of the field is as straightforward as in the vacuum case. In the case of superluminous motion, we can still use Eqs. (5) and (6) to derive the fields, being aware that we can have several retarded times for a single observation time: $t^{\prime}(t)$ is a multivalued function. For the superluminous case, Jefimenko's equation for the electric field is still valid, and can be written as

$$
\begin{aligned}
\mathbf{E}(\mathbf{x}, t)= & \frac{1}{4 \pi \epsilon} \int \mathrm{d}^{3} x^{\prime}\left[\frac{\hat{\mathbf{R}}}{R^{2}}\left[\rho\left(\mathbf{x}^{\prime}, t^{\prime}\right)\right]_{\mathrm{ret}}+\frac{\hat{\mathbf{R}}}{c_{n} R}\left[\frac{\partial \rho\left(\mathbf{x}^{\prime}, t^{\prime}\right)}{\partial t^{\prime}}\right]_{\mathrm{ret}}\right. \\
& \left.-\frac{1}{c_{n}^{2} R}\left[\frac{\partial \mathbf{J}\left(\mathbf{x}^{\prime}, t^{\prime}\right)}{\partial t^{\prime}}\right]_{\mathrm{ret}}\right],
\end{aligned}
$$

where $\hat{\mathbf{R}}=\frac{\mathbf{x}-\mathbf{x}^{\prime}}{\left|\mathbf{x}-\mathbf{x}^{\prime}\right|}$. Since the integrands are functions of $\mathbf{x}$, $\mathbf{x}^{\prime}$, and $t$ we can cast the following property:

$$
\left[\frac{\partial f\left(\mathbf{x}^{\prime}, t^{\prime}\right)}{\partial t^{\prime}}\right]_{\mathrm{ret}}=\frac{\partial}{\partial t}\left[f\left(\mathbf{x}^{\prime}, t^{\prime}\right)\right]_{\mathrm{ret}} \frac{\partial t\left(t^{\prime}, \mathbf{x}, \mathbf{x}^{\prime}\right)}{\partial t^{\prime}}=\frac{\partial}{\partial t}\left[f\left(\mathbf{x}^{\prime}, t^{\prime}\right)\right]_{\mathrm{ret}},
$$

which, along with the fact that $R=R\left(\mathbf{x}, \mathbf{x}^{\prime}\right)$ inside the integral (it is not a function of $t$ or $t^{\prime}$ ), allows us to rewrite Eq. (8) in the following way:

$$
\begin{aligned}
\mathbf{E}(\mathbf{x}, t)= & \frac{1}{4 \pi \epsilon}\left[\int \mathrm{d}^{3} x^{\prime} \frac{\hat{\mathbf{R}}}{R^{2}}\left[\rho\left(\mathbf{x}^{\prime}, t^{\prime}\right)\right]_{\mathrm{ret}}\right. \\
& +\frac{\partial}{\partial t} \int \mathrm{d}^{3} x^{\prime} \frac{\hat{\mathbf{R}}}{c_{n} R}\left[\rho\left(\mathbf{x}^{\prime}, t^{\prime}\right)\right]_{\mathrm{ret}} \\
& \left.-\frac{\partial}{\partial t} \int \mathrm{d}^{3} x^{\prime} \frac{1}{c_{n}^{2} R}\left[\mathbf{J}\left(\mathbf{x}^{\prime}, t^{\prime}\right)\right]_{\mathrm{ret}}\right] .
\end{aligned}
$$

We proceed now with the definition of our source. We consider a particle track, which is the building block for all 
Monte Carlo codes that calculate the electric field of an EAS within the microscopic approach. Let us consider a neutral atom or molecule in which all the particles are located approximately in the same point so that the charge density in all of space is 0 before the time $t_{1}$. At $t_{1}$, a point charge with charge $+q$ separates from the particle with charge $-q$ at the position $\mathbf{x}_{1}$. The $-q$ charge stays at the position $\mathbf{x}_{1}$, while the charge $+q$ travels in a straight line at constant speed until it is suddenly stopped at $t_{2}$, at the position $\mathbf{x}_{2}$. This charge density can be written as

$$
\begin{aligned}
\rho\left(\mathbf{x}^{\prime}, t^{\prime}\right)= & -q \delta^{3}\left(\mathbf{x}^{\prime}-\mathbf{x}_{1}\right) \Theta\left(t^{\prime}-t_{1}\right) \\
& +q \delta^{3}\left(\mathbf{x}^{\prime}-\mathbf{x}_{1}-\mathbf{v}\left(t^{\prime}-t_{1}\right)\right)\left[\Theta\left(t^{\prime}-t_{1}\right)-\Theta\left(t^{\prime}-t_{2}\right)\right] \\
& +q \delta^{3}\left(\mathbf{x}^{\prime}-\mathbf{x}_{2}\right) \Theta\left(t^{\prime}-t_{2}\right),
\end{aligned}
$$

where we have used the Dirac delta function $\delta$ and the Heaviside step function $\Theta$. The current density is, on the other hand,

$$
\mathbf{J}\left(\mathbf{x}^{\prime}, t^{\prime}\right)=q \mathbf{v} \delta^{3}\left(\mathbf{x}^{\prime}-\mathbf{x}_{1}-\mathbf{v}\left(t^{\prime}-t_{1}\right)\right)\left[\Theta\left(t^{\prime}-t_{1}\right)-\Theta\left(t^{\prime}-t_{2}\right)\right] .
$$

Equation (11) guarantees that charge is conserved. An electric field calculated with a nonconserved charge is not a solution of Maxwell's equations, since their definition implies the conservation of charge. Not taking into account the first and third terms in the rhs of Eq. (11) results in an unphysical radiation field, for instance (as shown in Sec. II C). Note that we have chosen a $-q$ charge in order to balance out the total charge contained in the space, but we could have used a single $+q$ charge for our problem, by substituting the first term in Eq. (11) by $+q \delta^{3}\left(\mathbf{x}^{\prime}-\mathbf{x}_{1}\right) \Theta\left(t_{1}-t^{\prime}\right)$, and the resulting electric field would only differ in a static Coulomb term.

The integrals containing a $\delta^{3}\left(\mathbf{x}^{\prime}-\mathbf{x}_{1}\right)$ or $\delta^{3}\left(\mathbf{x}^{\prime}-\mathbf{x}_{2}\right)$ are integrated trivially. However, the term $\delta^{3}\left(\mathbf{x}^{\prime}-\mathbf{x}_{1}-\mathbf{v}\left(t^{\prime}-t_{1}\right)\right)$ must be written as

$$
\begin{aligned}
\delta^{3}\left(\mathbf{g}\left(\mathbf{x}^{\prime}\right)\right) & =\delta^{3}\left(\mathbf{x}^{\prime}-\mathbf{x}_{1}-\mathbf{v}\left(t-\frac{\left|\mathbf{x}-\mathbf{x}^{\prime}\right|}{c_{n}}-t_{1}\right)\right) \\
& =\sum_{i} \frac{\delta^{3}\left(\mathbf{x}^{\prime}-\mathbf{x}_{p, i}(\mathbf{x}, t)\right)}{\left|\frac{\partial \mathbf{g}}{\partial \mathbf{x}^{\prime}}\right|_{\mathbf{x}_{p, i}}} \\
& =\sum_{i} \frac{\delta^{3}\left(\mathbf{x}^{\prime}-\mathbf{x}_{p, i}(\mathbf{x}, t)\right)}{\left|1-\mathbf{v} \cdot \hat{\mathbf{R}}\left(\mathbf{x}_{p, i}(\mathbf{x}, t)\right) / c_{n}\right|} \\
& =\sum_{i} \frac{\delta^{3}\left(\mathbf{x}^{\prime}-\mathbf{x}_{p, i}(\mathbf{x}, t)\right)}{\kappa_{i}}
\end{aligned}
$$

where $\mathbf{x}_{p, i}(\mathbf{x}, t)$ is the retarded position of the particle as a function of $(\mathbf{x}, t)$. These deltas are going to ensure that $\mathbf{x}^{\prime}$ is only evaluated at the retarded position, so upon integration we must substitute $\mathbf{x}^{\prime}$ for the retarded particle position and introduce the $\kappa$ factors, where we have defined $\kappa_{i}=\left|1-\mathbf{v} \cdot \hat{\mathbf{R}}\left(\mathbf{x}_{p, i}(\mathbf{x}, t)\right) / c_{n}\right|=\left|1-\mathbf{v} \cdot \hat{\mathbf{R}}_{i} / c_{n}\right|_{\text {ret }}$.

The index $i$ runs through the several possible retarded positions, that is, the roots of $\mathbf{g}\left(\mathbf{x}^{\prime}\right)=0 . \kappa$ is, in fact, related to the derivative of the observation time $t$ with respect to the retarded time $t^{\prime}$,

$$
\frac{\mathrm{d} t}{\mathrm{~d} t^{\prime}}=1-\mathbf{v} \cdot \hat{\mathbf{R}} / c_{n}
$$

Let $\theta$ be the angle formed by $\mathbf{v}$ and $\hat{\mathbf{R}}$, that is, the angle that the particle-observer line makes with the track. Let us define the Cherenkov angle $\theta_{C}$ as the angle that makes this derivative 0 .

$$
1-\mathbf{v} \cdot \hat{\mathbf{R}} / c_{n}=1-n \beta \cos \theta_{C}=0,
$$

that is,

$$
\theta_{C}=\operatorname{acos}\left(\frac{1}{n \beta}\right)
$$

where $\beta=v / c$. If, for our case, the track is always seen with an angle $\theta>\theta_{C}, \frac{\mathrm{d} t}{\mathrm{~d} t^{\prime}}>0$, so $t$ is monotonically increasing and there is only one $t^{\prime}$ for a given $t$. The same is true if $\theta<\theta_{C}$, although in this case the derivative is $\frac{\mathrm{d} t}{\mathrm{~d} t^{\prime}}<0$. However, if for a point along the track $\theta=\theta_{C}$ is verified, we can have two retarded times for a single observation time. Knowing this, we can drop the sum over $i$ and introduce the retardation brackets, keeping in mind that we may have two retarded positions below and above the Cherenkov angle whose fields we have to add.

Using Eq. (13) in Eq. (10) with the sources specified in Eqs. (11) and (12), the field can be written as

$$
\begin{aligned}
\mathbf{E}(\mathbf{x}, t)= & \frac{q}{4 \pi \epsilon}\left\{-\frac{\hat{\mathbf{R}}_{1}}{R_{1}^{2}}\left[\Theta\left(t^{\prime}-t_{1}\right)\right]_{\mathrm{ret}}-\frac{\hat{\mathbf{R}}_{1}}{c_{n} R_{1}} \frac{\partial}{\partial t}\left[\Theta\left(t^{\prime}-t_{1}\right)\right]_{\mathrm{ret}}\right. \\
& +\left[\frac{\hat{\mathbf{R}}}{\kappa R^{2}}\left(\Pi\left(t^{\prime}, t_{1}, t_{2}\right)\right)\right]_{\mathrm{ret}}+\frac{1}{c_{n}} \frac{\partial}{\partial t}\left[\frac{\hat{\mathbf{R}}}{\kappa R}\left(\Pi\left(t^{\prime}, t_{1}, t_{2}\right)\right)\right]_{\mathrm{ret}} \\
& -\frac{\mathbf{v}}{c_{n}^{2}} \frac{\partial}{\partial t}\left[\frac{1}{\kappa R}\left(\Pi\left(t^{\prime}, t_{1}, t_{2}\right)\right)\right]_{\mathrm{ret}} \\
& \left.+\frac{\hat{\mathbf{R}}_{2}}{R_{2}^{2}}\left[\Theta\left(t^{\prime}-t_{2}\right)\right]_{\mathrm{ret}}+\frac{\hat{\mathbf{R}}_{2}}{c_{n} R_{2}} \frac{\partial}{\partial t}\left[\Theta\left(t^{\prime}-t_{2}\right)\right]_{\mathrm{ret}}\right\},
\end{aligned}
$$

having defined the boxcar function

$$
\Pi\left(t^{\prime}, t_{1}, t_{2}\right)=\Theta\left(t^{\prime}-t_{1}\right)-\Theta\left(t^{\prime}-t_{2}\right)
$$

and $R_{1,2}, \hat{\mathbf{R}}_{1,2}$ being the distances and unit vectors from the first and last point of the track to the observer. In order to simplify the expression, we can derive the terms $\frac{\partial}{\partial t}\left[\Theta\left(t^{\prime}-t_{i}\right)\right]_{\mathrm{ret}}$. The step function is always 0 or 1 , either using retarded time or observation time, so its derivative 
with respect to observation time must be a unit Dirac delta, but at the observation time $t$ corresponding to the retarded time $t^{\prime}=t_{i}$

$$
\frac{\partial}{\partial t}\left[\Theta\left(t^{\prime}-t_{i}\right)\right]_{\mathrm{ret}}=\left[\frac{\partial}{\partial t^{\prime}} \Theta\left(t^{\prime}-t_{i}\right)\right]_{\mathrm{ret}}=\left[\delta\left(t^{\prime}-t_{i}\right)\right]_{\mathrm{ret}},
$$

which allows us to rewrite Eq. (18) as

$$
\begin{aligned}
\mathbf{E}(\mathbf{x}, t)= & \frac{q}{4 \pi \epsilon}\left\{-\frac{\hat{\mathbf{R}}_{1}}{R_{1}^{2}} \Theta\left(t-t_{1}-R_{1} / c_{n}\right)-\frac{\hat{\mathbf{R}}_{1}}{c_{n} R_{1}} \delta\left(t-t_{1}-R_{1} / c_{n}\right)+\left[\frac{\hat{\mathbf{R}}}{\kappa R^{2}}\left(\Pi\left(t^{\prime}, t_{1}, t_{2}\right)\right)\right]_{\mathrm{ret}}+\frac{1}{c_{n}} \frac{\partial}{\partial t}\left[\frac{\hat{\mathbf{R}}}{\kappa R}\left(\Pi\left(t^{\prime}, t_{1}, t_{2}\right)\right)\right]_{\mathrm{ret}}\right. \\
& \left.-\frac{\mathbf{v}}{c_{n}^{2}} \frac{\partial}{\partial t}\left[\frac{1}{\kappa R}\left(\Pi\left(t^{\prime}, t_{1}, t_{2}\right)\right)\right]_{\mathrm{ret}}+\frac{\hat{\mathbf{R}}_{2}}{R_{2}^{2}} \Theta\left(t-t_{2}-R_{2} / c_{n}\right)+\frac{\hat{\mathbf{R}}_{2}}{c_{n} R_{2}} \delta\left(t-t_{2}-R_{2} / c_{n}\right)\right\} .
\end{aligned}
$$

We have used the fact that $t^{\prime}=t-R_{i} / c_{n}$ before $t^{\prime}=t_{1}$ and after $t^{\prime}=t_{2}$. The first line in Eq. (21) contains a static Coulomb field turned on at $t_{1}$ and an impulse radiation field. The third line shows a similar field, but corresponding to the instant when the particle stops. The second line is analogous to the Heaviside-Feynman expression of the electric field of a particle existing for a limited time, used in [32] for the calculation of the electric field. The impulse fields with the form

$$
\frac{\hat{\mathbf{R}}_{i}}{c_{n} R_{i}} \delta\left(t-t_{i}-R_{i} / c_{n}\right)
$$

come directly from the imposition of charge conservation, and they represent a radiation field that would be missing had we not taken into account a realistic charge density. This field is created by the changes in the charge density, and so we can pair it with the second term in the second line of Eq. (21), since they must always appear together. Let us develop what happens at the time $t_{1, \mathrm{obs}}=t_{1}+R_{1} / c_{n}$ with these two terms,

$$
\begin{aligned}
\mathbf{E}_{\text {cons }}(\mathbf{x}, t)= & \frac{q}{4 \pi \epsilon c_{n}}\left[-\frac{\hat{\mathbf{R}}_{1}}{R_{1}}+\frac{\hat{\mathbf{R}}_{1}}{R_{1}\left|1-\mathbf{v} \cdot \hat{\mathbf{R}}_{1} / c_{n}\right|}\right] \\
& \times \delta\left(t-t_{1}-R_{1} / c_{n}\right) .
\end{aligned}
$$

The second term on the rhs in Eq. (23) cannot exist on its own. It represents a sudden appearance of charge, which is not possible without a creation of an opposite charge at the same point, which in turns creates an electric field given by the first term. Analogously for the deceleration of our particle, we need to have a field of the kind

$$
\begin{aligned}
\mathbf{E}_{\text {cons }}(\mathbf{x}, t)= & \frac{q}{4 \pi \epsilon c_{n}}\left[\frac{\hat{\mathbf{R}}_{2}}{R_{2}}-\frac{\hat{\mathbf{R}}_{2}}{R_{2}\left|1-\mathbf{v} \cdot \hat{\mathbf{R}}_{2} / c_{n}\right|}\right] \\
& \times \delta\left(t-t_{2}-R_{2} / c_{n}\right),
\end{aligned}
$$

where the first term on the rhs is reminding us that the particle does not disappear but instead stops and stays at the same place.

\section{B. Far-field approximation. The ZHS formula}

Equation (21) can be taken to the far-field limit after which we arrive at the time-domain ZHS formula [35]. We show in Appendix A how to retrieve the ZHS formula,

$$
\mathbf{E}_{\mathrm{ZHS}}(\mathbf{x}, t)=-\frac{e \mu_{r}}{4 \pi \epsilon_{0} c^{2} R} \mathbf{v}_{\perp} \frac{\delta\left(t-\frac{n R}{c}-(1-n \beta \cos \theta) t_{1}\right)-\delta\left(t-\frac{n R}{c}-(1-n \beta \cos \theta) t_{2}\right)}{1-n \beta \cos \theta}
$$

\section{Calculation of time-domain field and comparison with far-field (ZHS) formula}

Let us consider an electron track $(q=-e)$ along with a positive charge $(q=e)$ that stays at rest. The time-domain electric field of such a configuration can be calculated numerically using Eq. (21). Even though one of the main aims of this work is to develop an equation to calculate the field created by an extensive air shower, Eq. (21) is valid for dense dielectric media as well. Because of this, and also the fact that superluminous effects (like the Cherenkov cone) are more prominent in dense media, we choose a dielectric medium with a refractive index of $n=1.78$, akin to that of deep Antarctic ice, to perform our calculations and to better illustrate our point. Let us embed a $1.2 \mathrm{~m}$ long electron 


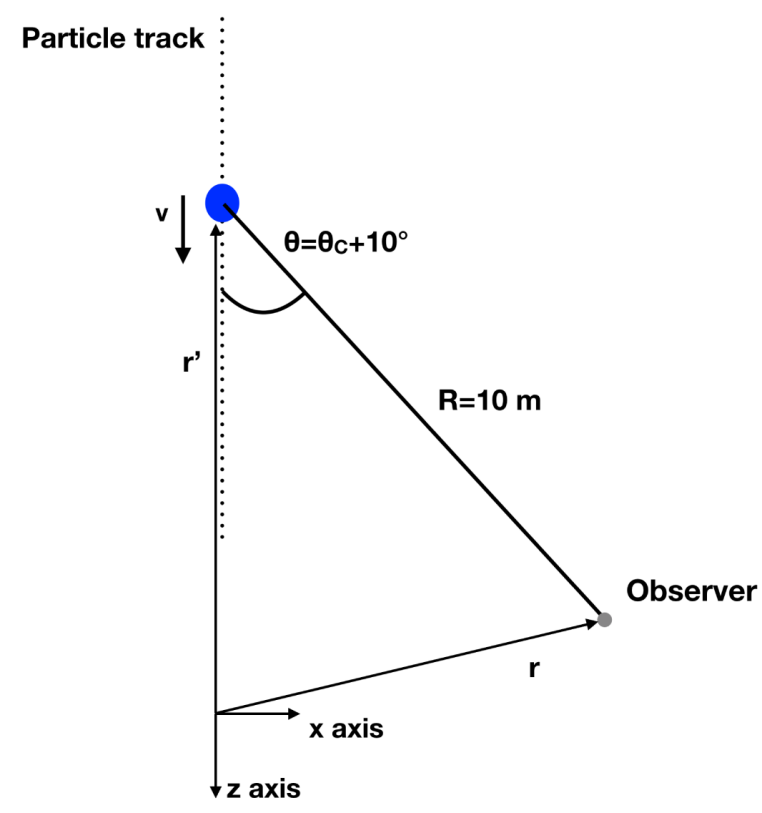

FIG. 1. Sketch containing the particle track and the observer. $\theta$ is the angle formed by the particle velocity $\mathbf{v}$ and the line joining the particle and the observer. The observer lies at $\mathbf{r}$ and the particle at $\mathbf{r}^{\prime}$. $R$ is the distance between observer and particle. The particle travels along the $z$ axis (indicated in the figure along with the $x$ axis).

track traveling along the $z$ axis with a speed of $v \sim c$ in the aforementioned medium and place an observer at $10 \mathrm{~m}$ and at an angle of $\theta_{C}+10^{\circ}=65.82^{\circ}$, with $\theta_{C}$ being the Cherenkov angle (see Fig. 1). In order to evaluate Eq. (24), we choose a numerical step $\Delta t=0.05 \mathrm{~ns}$ and define our numerical derivative at a point $t_{i}$ as a two-point derivative as follows:

$$
\frac{\mathrm{d} f}{\mathrm{~d} t}\left(t_{0}\right) \approx \frac{f\left(t_{0}+\Delta t\right)-f\left(t_{0}-\Delta t\right)}{2 \Delta t} .
$$

Besides evaluating it, we can compare our formula with the ZHS algorithm, which has been proven [37] to give the correct results for the far-field regime, that is, $k R \gg 1$, with $k$ being the wave number and $R$ the distance between particle and observer. We show the results in Fig. 2. We find that the ZHS algorithm (blue points) and Eq. (21) (red line) give the same result, which is understandable knowing that the field lasts for several nanoseconds, which means that for the frequencies contributing the most to the radiation field, $k R \sim 2 \pi \frac{R}{c_{n} T} \sim 370$, with $T$ being the inverse of the frequency $\nu$, of the order of GHz. However, if we do not take into account the terms in Eqs. (23) and (24) that guarantee the conservation of charge, we are not able to reproduce the impulses corresponding to the acceleration and deceleration of the particle (black lines), meaning that such terms cannot be omitted for a physical calculation of the electric field. Putting an observer at $100 \mathrm{~m}$ from the track, where the acceleration and deceleration fields are more important

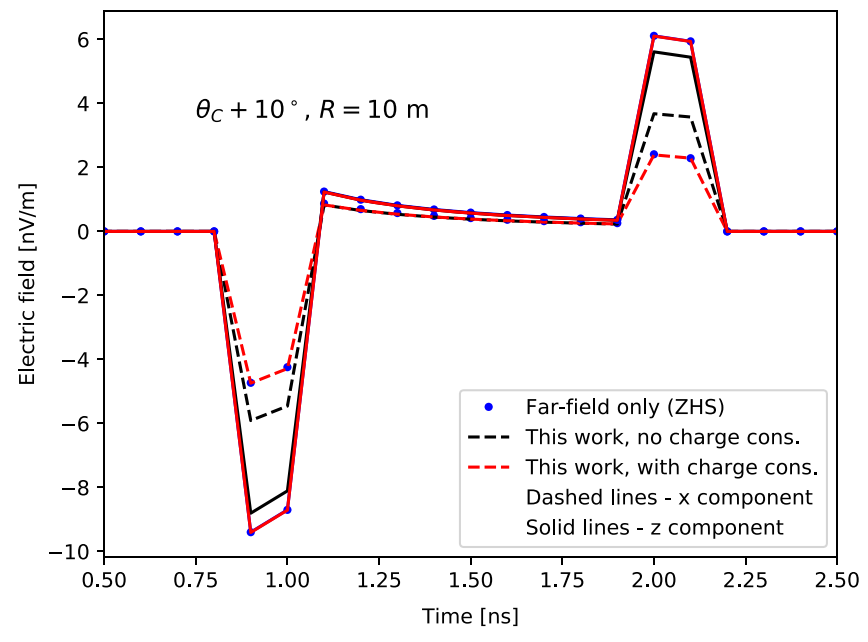

FIG. 2. Electric field as a function of time created by a $1.2 \mathrm{~m}$ long electron track traveling along the $z$ axis in deep Antarctic ice $(n=1.78)$. The observer is placed at $\theta_{C}+10^{\circ}$ and at a distance of $R=100 \mathrm{~m}$. Red lines represent Eq. (21), blue points represent the ZHS algorithm, and black lines represent Eq. (21) but without the terms needed for the conservation of charge. The numerical time step is $\Delta t=0.1 \mathrm{~ns}$.

than in the previous case, demonstrates that not including this term results in an incorrect impulse field; see Fig. 3.

We show in Fig. 4 that our formula is also valid for calculating the field inside the Cherenkov cone. The predicted polarity is in agreement with the polarity from the ZHS formula. Besides, outside (Fig. 2) and inside the Cherenkov (Fig. 4) cone we find the same polarity. Let us remember that the acceleration and deceleration fields are interchanged when going from outside the cone to inside the cone, meaning that inside the cone the observer sees the field from the deceleration first. Figure 4 illustrates the narrowing of the electric field as the observer moves closer to the Cherenkov angle.

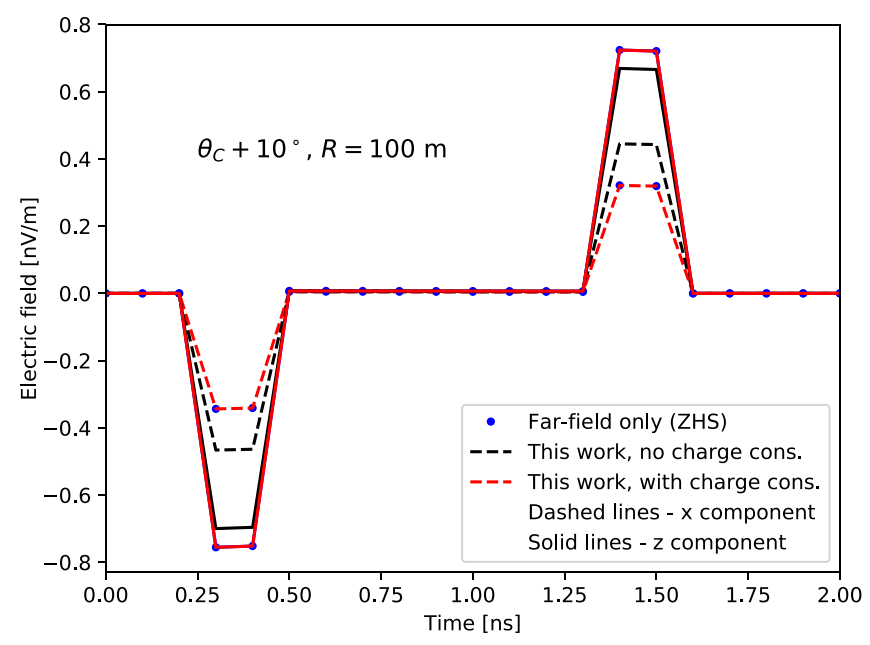

FIG. 3. Same as Fig. 2 but with an observer at $R=100 \mathrm{~m}$. 


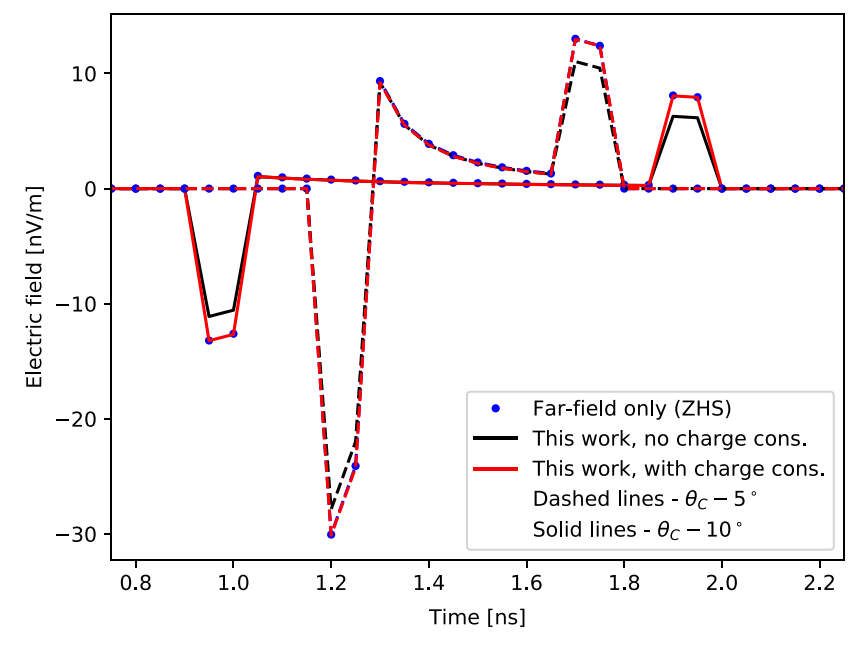

FIG. 4. Same as Fig. 2 but only the $x$ component of the field and with an observer at $\theta_{C}-10^{\circ}$ (solid lines) and another one at $\theta_{C}-5^{\circ}$ (dashed lines).

Now that we have shown that our formula yields sensible results in time domain, we proceed with the study of its frequency-domain counterpart.

\section{ELECTRIC FIELD OF A PARTICLE TRACK IN FREQUENCY DOMAIN}

We have two ways to know the emission of a particle track in frequency domain. Either we transform Eq. (21) (see Appendix B) or we solve Maxwell's equations in frequency domain (see Appendix C). Both approaches give the following equation:

$$
\begin{aligned}
\mathbf{E}(\mathbf{x}, \omega)= & \frac{q}{4 \pi \epsilon}\left\{-\frac{\hat{\mathbf{R}}_{1}}{R_{1}} e^{i \omega t_{1}} e^{i k R_{1}}\left[\frac{i}{\omega R_{1}}+\frac{1}{c_{n}}\right]\right. \\
& +\int_{t_{1}}^{t_{2}} \mathrm{~d} t^{\prime} e^{i \omega t^{\prime}} e^{i k R}\left[\frac{\hat{\mathbf{R}}}{R^{2}}-\frac{i \omega}{c_{n}} \frac{\hat{\mathbf{R}}}{R}+\frac{i \omega}{c_{n}^{2}} \frac{\mathbf{v}}{R}\right] \\
& \left.+\frac{\hat{\mathbf{R}}_{2}}{R_{2}} e^{i \omega t_{2}} e^{i k R_{2}}\left[\frac{i}{\omega R_{2}}+\frac{1}{c_{n}}\right]\right\} .
\end{aligned}
$$

\section{A. Calculation of frequency-domain field and comparison with far-field (ZHS) formula}

Another frequency-domain equation for the same problem of a track traveling along the $z$ axis can be found in [37]. The radial $(\rho)$ and vertical $(z)$ components of the electric field can be written in this work's notation as follows:

$$
\begin{aligned}
E_{\rho}(\mathbf{x}, \omega)= & i \frac{q v}{\omega} \frac{1}{4 \pi \epsilon} \int_{t_{1}}^{t_{2}} \mathrm{~d} t^{\prime} e^{i \omega t^{\prime}} \frac{e^{i k R}}{R} \\
& \times \sin \theta \cos \theta\left[b\left(b-\frac{1}{R}\right)+\frac{1}{R^{2}}\right],
\end{aligned}
$$

and

$$
\begin{aligned}
E_{z}(\mathbf{x}, \omega)= & i \frac{q v}{\omega} \frac{1}{4 \pi \epsilon} \int_{t_{1}}^{t_{2}} \mathrm{~d} t^{\prime} e^{i \omega t^{\prime}} \frac{e^{i k R}}{R} \\
& \times\left[b^{2} \cos ^{2} \theta+\frac{\cos ^{2} \theta}{R^{2}}-\frac{b}{R}\left(\cos ^{2} \theta-1\right)\right] \\
& +i \omega \frac{\mu_{0}}{4 \pi} q v \int_{t_{1}}^{t_{2}} \mathrm{~d} t^{\prime} e^{i \omega t^{\prime}} \frac{e^{i k R}}{R},
\end{aligned}
$$

where $b\left(t^{\prime}\right)=i k-\frac{1}{R}$. Equations (28) and (29) have been derived following the same premises as Eq. (B9), but circumventing the problem of charge conservation by explicitly using the Lorenz gauge condition in frequency domain. This way, it is possible to calculate the scalar potential $\Phi$ by means of the vector potential $\mathbf{A}$, that is,

$$
\mathbf{A}(\mathbf{x}, \omega)=i \epsilon \mu_{0} \omega \Phi(\mathbf{x}, \omega),
$$

allowing us to arrive at a correct field without having to write a correct charge density. In exchange, we arrive at a more cumbersome electric field [Eqs. (28) and (29)] than in the present work [Eq. (27)], although both expressions are equivalent at nonzero frequency.

We show an example of this equivalence in Fig. 5, where both approaches give the same numerical result for an observer placed at the Cherenkov angle of a track. As a consequence, Eq. (27) contains the exact field of a track, including the Cherenkov shock wave [38]. In particular, this means that in far-field regime, Eq. (27) agrees with the ZHS algorithm for large frequencies $(k R \gg 1)$, which is shown in Fig. 5.

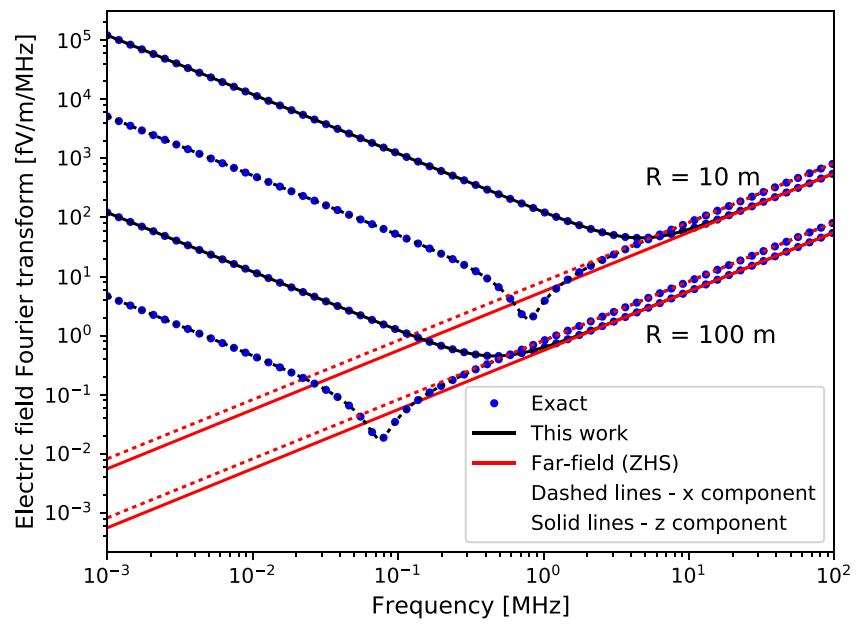

FIG. 5. Fourier transform of the electric field ( $x$ and $z$ components) as a function of frequency for a $1.2 \mathrm{~m}$ long track traveling in deep Antarctic ice $(n=1.78)$ along the $z$ axis at a speed $v \approx c$. The observer is placed at the Cherenkov angle, at a distance of $R=10 \mathrm{~m}$ and $R=100 \mathrm{~m}$. The exact field from Eqs. (28) and (29) (blue points) is plotted, along with the field from Eq. (27) (black lines) and the field calculated with the ZHS algorithm (red lines). The first two approaches are indistinguishable, and the third one agrees with them in the far-field regime. 
The advantage of the present work with respect to the formalism in [37] is that in this work we have derived the time-domain field, which was not present in [37]. The timedomain field is vastly superior in terms of computing power to its frequency-domain counterpart, since we do not have the need to perform numerical integration. Besides, the obtention of the time-domain picture is already desirable in itself for reasons of physical insight. In this work we have also presented a frequency-domain formula that is simpler than the formula in [37], and we have proven that it can be obtained directly in frequency domain and as a transform of the time-domain version. The present work's frequencydomain formula is equivalent at nonzero frequency to the formulas in [37], because they are obtained from the same potentials, although expressed in a different fashion. In [37] the Lorenz gauge condition is explicitly used to derive the scalar potential from the vector potential, which instead leads us to more complicated formulas that are harder to transform to time domain and that do not contain the correct description of the field at zero frequency (there are some delta functions missing), which is necessary for this transform.

\section{IMPLEMENTATION OF THE TIME-DOMAIN FORMULA IN THE SELFAS MONTE CARLO CODE}

Once it has been shown that Eq. (21) is an adequate formula for the description of the electric field created by a particle track, we can embed this formula in an air shower simulation code in order to calculate the electric field generated by an extensive air shower. We have chosen the SELFAS [32] MC code. SELFAS uses a hybrid approach for the simulation-a given number of particles and their properties are sampled from probability distributions (longitudinal and lateral positions, velocity, etc.) and then each particle is subject to interactions and microscopically followed for an atmospheric depth of $15 \mathrm{~g} / \mathrm{cm}^{2}$. The combination of macroscopic particle distributions and microscopic treatment of each individual particle constitutes what we call the hybrid approach. Currently, SELFAS samples the particles using the longitudinal shower profile given by CONEX [39], a lightweight code that produces results compatible with the full MC CORSIKA. SELFAS has been recently upgraded with a state-of-the-art treatment of the atmosphere with its inclusion of the Global Data Assimilation System [40,41].

Before we implement Eq. (21) in the code, special attention has to be paid to the Coulomb terms of the static particles left behind, since they are not detectable in an EAS, first, because the physical situation is not the same. If a random track inside the shower is picked, there is a high probability that this track is the continuation of an ongoing particle trajectory and not the beginning, meaning there is no opposite charge left behind and its static field can be ignored. Analogously, a randomly chosen track will likely not be the end of the true particle trajectory, so we do not suppose that the particle rests at the end of the track and ignore its Coulomb field. Secondly, if there are static charged particles left behind, the medium itself changes in order to regain electrostatic equilibrium and minimize the static electric fields. Because of these physical reasons, we do not compute the Coulomb terms $\frac{\hat{\mathbf{R}}_{1,2}}{R_{1,2}^{2}} \Theta\left(t-t_{1,2}-R_{1,2} / c_{n}\right)$.

One way of justifying this approximation is to calculate an estimation for this electrostatic emission with a simple model. Let us assume a vertical $10^{18} \mathrm{eV}$ shower with a number of charged particles $N_{\text {particles }} \sim 10^{9}$. For each charged particle, there is a particle of the opposite sign in the shower or in the surrounding atmosphere, ${ }^{1}$ but we assume that all the electrostatic emission comes from the excess charge of the shower ( $\sim 20 \%$ of the total number of particles) and that all the particles are located at the shower maximum, at a height of $h \sim 4 \mathrm{~km}$. We also assume that the electrostatic field is turned on at a time $t_{0}$, making the contribution of the whole shower coherent at all frequencies, and we treat it as constant, effectively ignoring ion recombination. The radial component of the electrostratic field can be written as

$$
E_{\text {static }} \sim \frac{e \Delta q N_{\text {particles }}}{4 \pi \epsilon_{0} h^{2}} \Theta\left(t-t_{0}\right)
$$

$\Delta q \sim 0.2$ is the magnitude of the excess charge. This translates into the frequency domain at nonzero frequency as

$$
E_{\text {static }}(\omega) \sim \frac{e \Delta q N_{\text {particles }}}{4 \pi \epsilon_{0} h^{2}} \frac{i}{\omega},
$$

which decreases with frequency. We can thus obtain an estimation for the field from the Coulomb terms taking as a reference the field at $1 \mathrm{MHz}$,

$$
E_{\text {static }}(\nu) \sim 2.9 \frac{1 \mathrm{MHz}}{\nu} \mathrm{nV} \mathrm{m}^{-1} \mathrm{MHz}^{-1},
$$

which is negligible for our calculations. The actual field should be lower, due to the recombination of the ions.

Another important matter is what to do with the particles that reach the ground, since our calculation is valid for a single medium. When a track reaches ground, its field can be calculated as the sum of the field of a track that stops just above the ground and another one accelerating at the same time just below the ground, and making their distance as small as possible. This approach explains transition radiation $[42,43]$. With this scheme in mind, we can separate the total field into three different contributions:

\footnotetext{
${ }^{1}$ Actually, if the atmosphere is neutral, there is always a small excess of positive charge given by the atomic number of the primary cosmic ray.
} 
(i) The direct field coming from the track above the surface (as if there were no boundary). This contribution can be calculated, at all frequencies, with Eq. (21).

(ii) The field created by the response of the interface (in this case, the ground) to the field emitted by the track above the surface. If the track lies in the far field with respect to the ground, and also the antenna lies in the far field with respect to the ground, that is, if the distances to the ground are much larger than the observation wavelength, we can use geometrical optics and the standard Fresnel coefficients. If the antenna is close to the ground but the track is in the far field with respect to the ground, a possible workaround is to use the reciprocity theorem $[44,45]$, which allows us to know the antenna voltage with the far-field antenna pattern and the direct field of the track.

In our case, since we are interested in frequencies around the $\mathrm{MHz}(\lambda \sim 300 \mathrm{~m})$, and there is a nonnegligible number of particles arriving at ground, we cannot use geometrical optics. Moreover, the antennas are placed close to the ground in most radio EAS experiments $(9 \mathrm{~m}$ high in the case of EXTASIS [11]). When the emitter and receiver are in the near field of the ground, what we have is a wave that is a mixture of a surface wave and a reflected (in the sense of geometrical optics) wave [46], which can be more similar to the former or the latter depending on the actual physical configuration. The calculation of such a field lies outside of the scope of the present paper, so as a first-order approximation we do not calculate this contribution. Nevertheless, the importance of this contribution constitutes an interesting study that we are currently carrying out.

(iii) The contribution of the underground track is ignored. Because of the larger attenuation losses of radio waves inside soil [47], we expect this contribution to be much smaller than the surface wave contribution.

To sum up with, we calculate the direct contribution from the parts of the track above the ground. This is equivalent to suddenly stopping the particles upon their arrival to the ground, which seems reasonable, since these electrons are in fact beta radiation with a mean speed of $\sim 0.3 c$ and they are stopped in $<1 \mathrm{~cm}$ of soil.

\section{SIMULATIONS FOR EAS AND COMPARISON WITH THE FAR-FIELD EMISSION}

We have simulated a proton-induced $1 \mathrm{EeV}$ shower, with a zenith angle of $30^{\circ}$ and coming from the east $\left(\phi=0^{\circ}\right.$ marks the east and $\phi=90^{\circ}$ the north), using $2 \times 10^{8}$ particles. The ground lies at an altitude of $1400 \mathrm{~m}$, as is the case for the Pierre Auger Observatory. Several antennas have been placed north of the shower core, at different
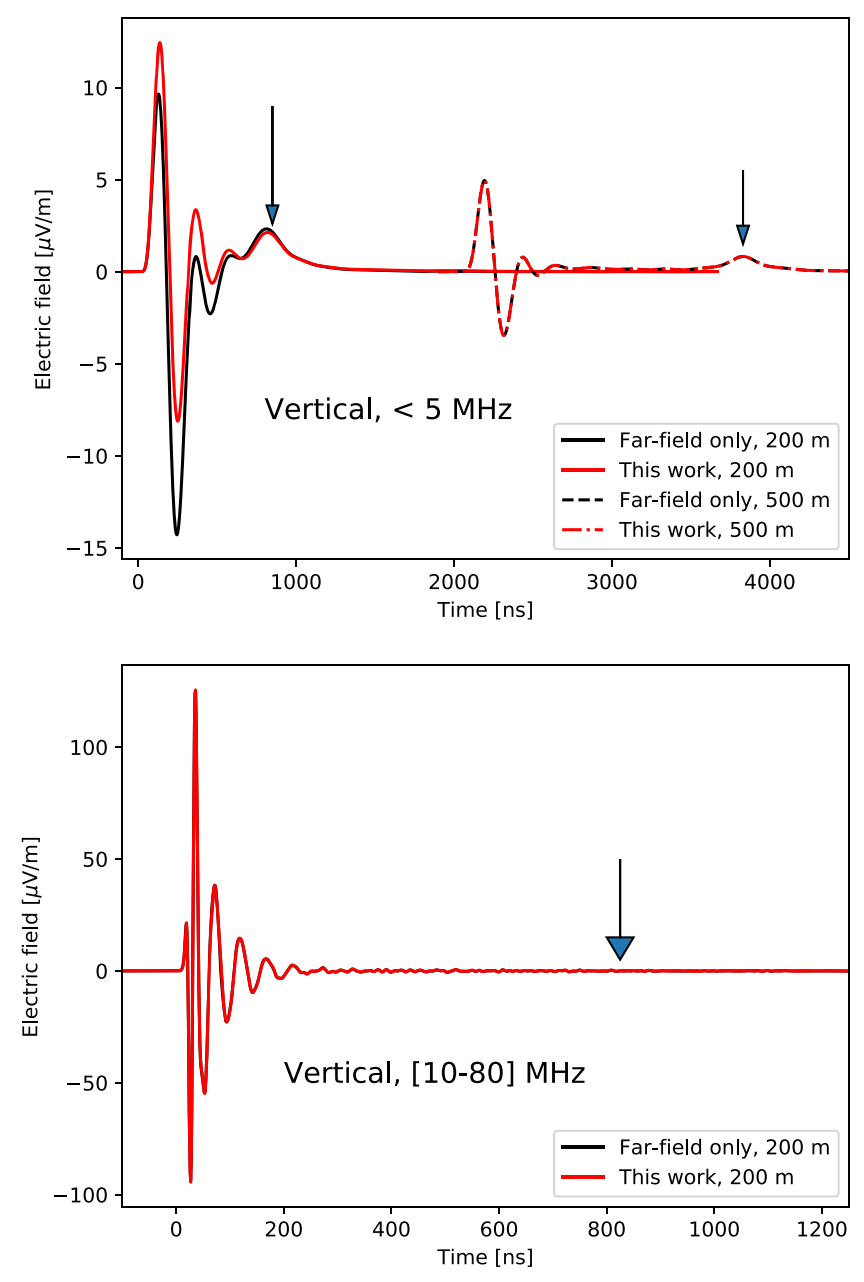

FIG. 6. Electric field as a function of time created by a $1 \mathrm{EeV}$ proton-induced shower with $30^{\circ}$ of zenithal angle and coming from the east $\left(\phi=0^{\circ}\right)$. Times have been arbitrarily offset. Traces have been numerically transformed to frequency, then filtered with a sixth-order low-pass Butterworth filter and transformed back to time domain. This work's formula (red lines) and the farfield approximation (ZHS, black lines) are plotted. Observers have been placed at 200 (solid lines) and $500 \mathrm{~m}$ (dashed lines) east from the shower core. The sudden death field (indicated by the arrows) is visible after the principal pulse in each trace below $5 \mathrm{MHz}$. Top: $5 \mathrm{MHz}$ low-pass filter. Bottom: 10-80 MHz bandpass filter. See text for details.

distances from it. Since Eq. (21) is equivalent to the ZHS formula in the far field, we focus on the low-frequency part of the electric field and filter our resulting electric fields with a low-pass (or band-pass, depending on the case) sixth-order Butterworth filter. ${ }^{2}$ We show in Fig. 6 the resulting vertical component of the electric field (filtered

\footnotetext{
${ }^{2}$ A Butterworth filter has the important property of preserving causality. An unphased step or box filter gives an acausal behavior. In particular, for a typical EAS signal, it produces an unphysical ringing with a nonzero field well before the actual arrival time of the wave at the observing location.
} 


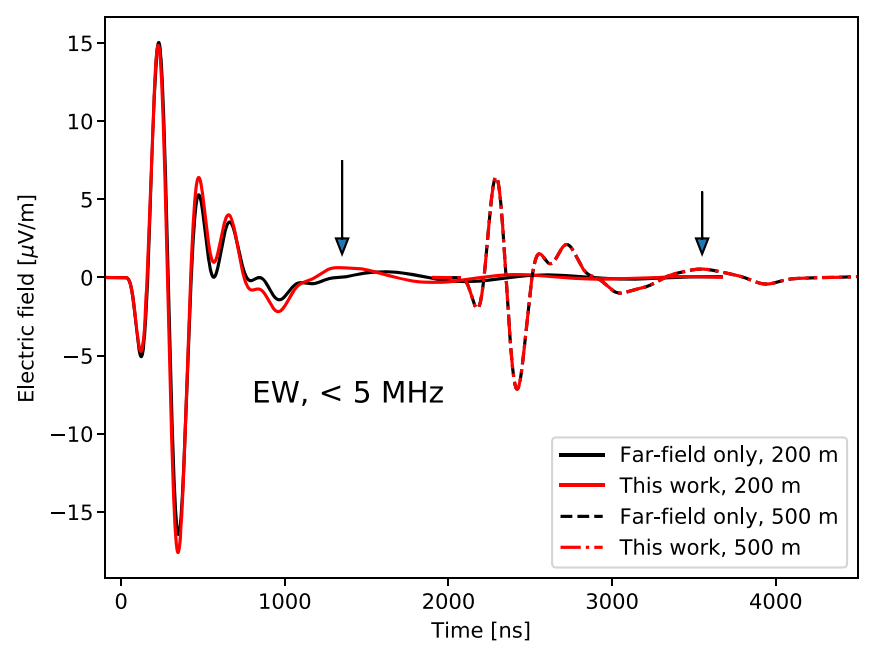

FIG. 7. Same as Fig. 6, top, but for the east-west (EW) polarization. See text for details.

at frequencies $<5 \mathrm{MHz}$ ) for one observer at 200 m east of the shower core and another at $500 \mathrm{~m}$. The electric fields have been calculated with the far field (ZHS) and complete approach [Eq. (21)] using the very same shower. It is worth noting that at low frequency (Fig. 6, top, and Fig. 7) and at a distance of $200 \mathrm{~m}$, differences between the far field and the complete approach are quite important, whereas for $500 \mathrm{~m}$ both approaches are visually indistinguishable. This is also the case when filtering between [20-80] $\mathrm{MHz}$ for the observer at $200 \mathrm{~m}$ (Fig. 6, bottom). Another important feature found is the existence of a pulse originated by the sudden deceleration of the shower front, marked by the arrows in Fig. 6. Note that this deceleration is coherent only at low frequencies, since the pulse disappears filtering in the [20-80] MHz band. From now on, we call this pulse the SDP [26].

In Fig. 7 we show the geomagnetic component (EW), finding that at low frequencies, the main geomagnetic pulse from the EAS is changed in a non-negligible way at $200 \mathrm{~m}$ from the shower core. This is a remarkable fact, because the altitude of the shower maximum being at several kilometers, one could think that the far-field approach could suffice for computing near the shower axis at $\sim \mathrm{MHz}$ frequencies, but this is not the case.

We show in Fig. 8 some examples of the spectrum amplitude of the electric field for the far-field and complete approaches. For an observer at $500 \mathrm{~m}$, differences between the two approaches are negligible, whereas at $200 \mathrm{~m}$ and below $10 \mathrm{MHz}$ differences are quite important. In order to quantify them, we show the relative difference between the two approaches for the same shower in Fig. 9, defined as the absolute value of the difference between the far field and the exact formula divided by the amplitude of the exact formula (for a given polarization). Below $10 \mathrm{MHz}$, and for this given shower, both approaches differ considerably and one should use the exact formula if a complete electric field

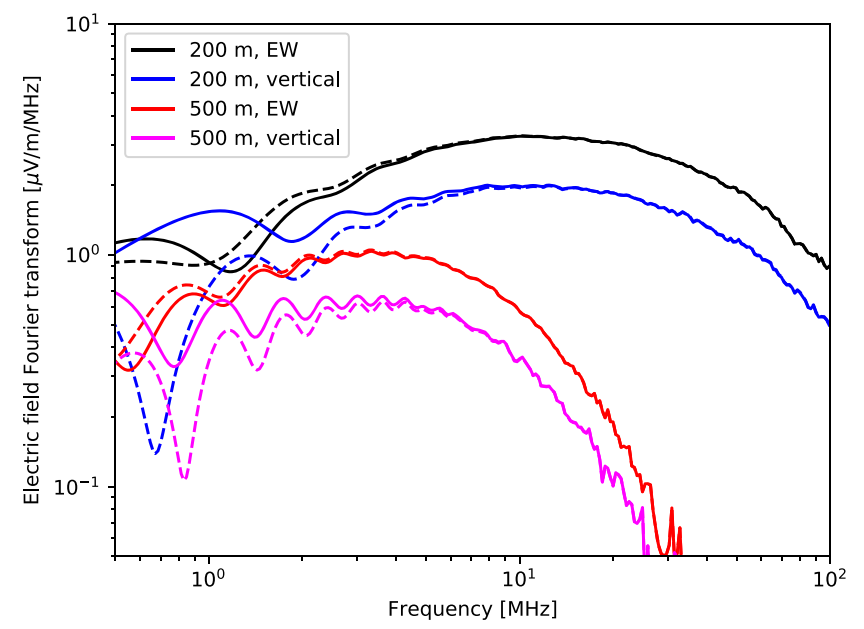

FIG. 8. Amplitude of the Fourier transform of the electric field as a function of frequency created by a $1 \mathrm{EeV}$ proton-induced shower with $30^{\circ}$ of zenithal angle and coming from the east $\left(\phi=0^{\circ}\right)$. Dashed lines indicate the result of the complete formula, while solid lines portray the far-field calculation. We have plotted the east-west and vertical polarizations for an observer at $200 \mathrm{~m}$ and another at $500 \mathrm{~m}$ north of the shower core.

is needed. The relative difference falls with frequency until $\sim 10 \mathrm{MHz}$, where it begins to be dominated by the incoherent emission of the particles of the lower part of the shower that are closer to the observer (located in the near field). The error lies around $\sim 1 \%$ above $10 \mathrm{MHz}$, except for the vertical component at $50 \mathrm{~m}$ from the shower core, where the discrepancy between the two formulas is still large, up to $\sim 50 \mathrm{MHz}$.

So as to elucidate whether we should use the exact formula instead of the far-field approximations for most of

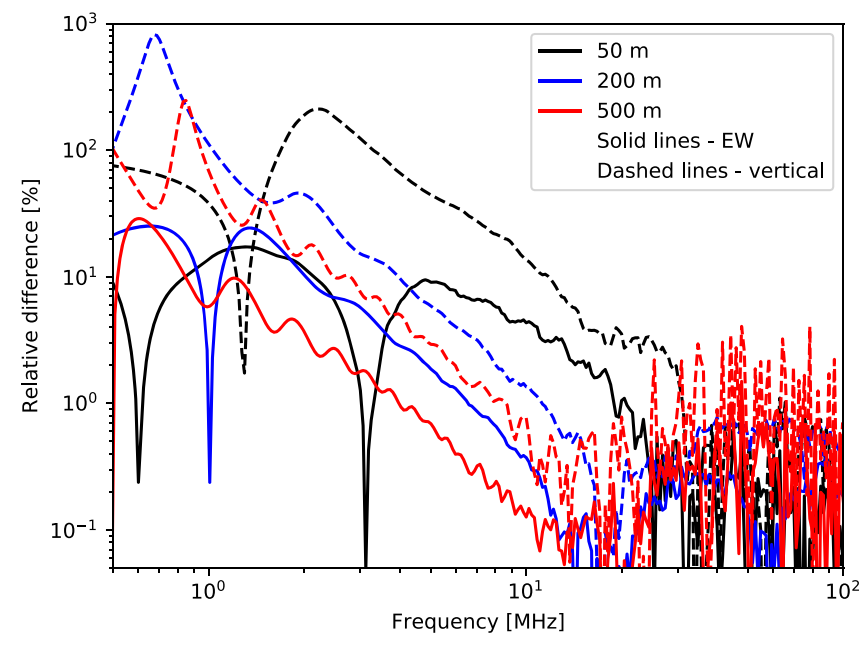

FIG. 9. Relative difference as a function of frequency between the exact solution and the far-field approximation (ZHS) for the same shower of Fig. 8. We have placed observers at 50 (black), 200 (blue), and $500 \mathrm{~m}$ (red). Solid lines indicate EW polarization and dashed lines vertical polarization. 

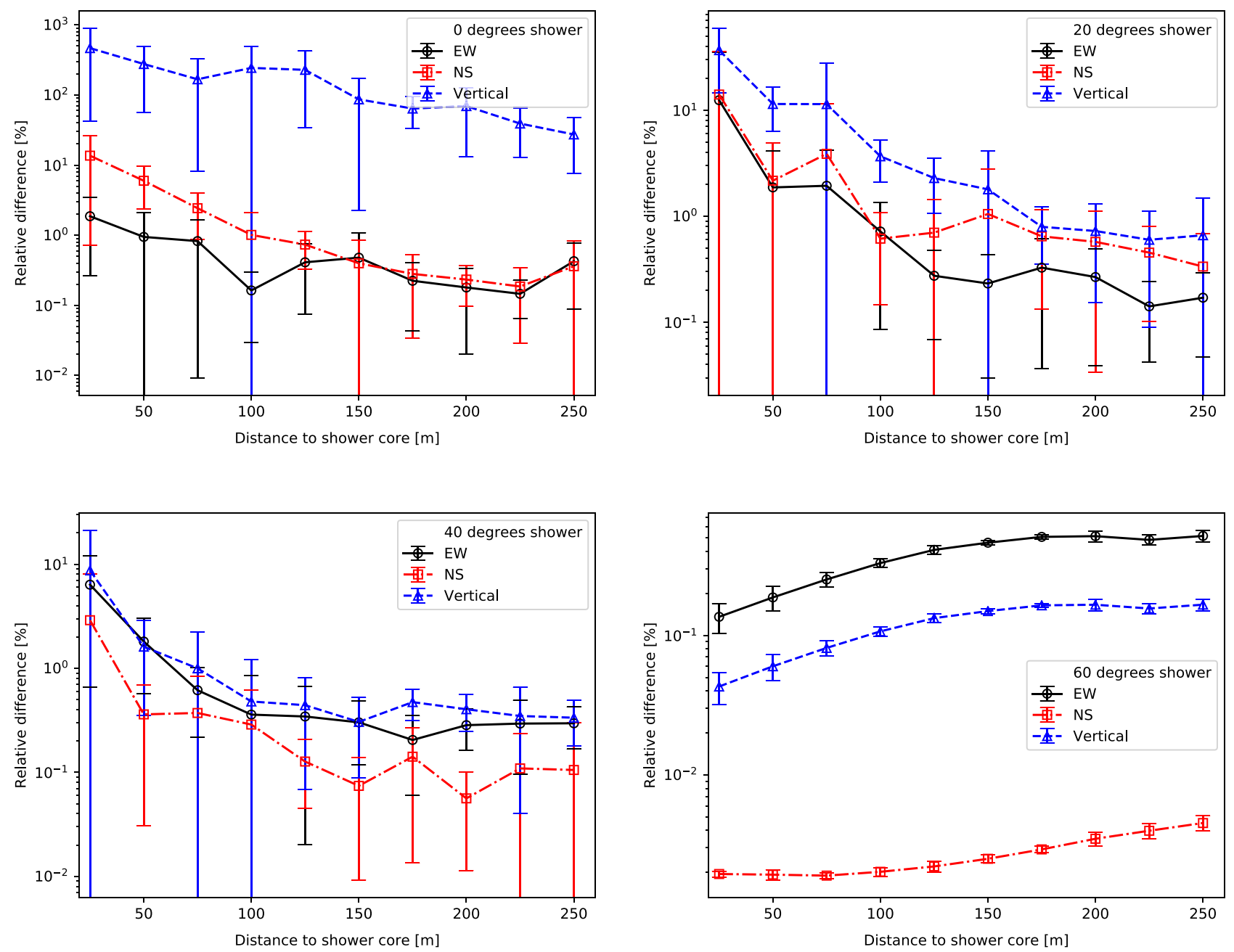

FIG. 10. Relative difference as a function of distance between the exact solution and the far-field approximation (ZHS) for the same shower of Fig. 8. The relative differences have been calculated for $1 \mathrm{EeV}$ showers at frequency of $20 \mathrm{MHz}$ and averaged over ten showers. The means of these differences are represented by the marks, while the error bars indicate the standard deviation. EW, NS, and vertical polarizations are plotted as indicated in the legend. Top left: $0^{\circ}$ zenith angle shower. Top right: $20^{\circ}$ zenith angle shower. Bottom left: $40^{\circ}$ zenith angle shower. Bottom right: $60^{\circ}$ zenith angle shower.

today's radio experiments, which measure the electric field above $20 \mathrm{MHz}$, we have computed the relative difference as a function of the distance for a fixed frequency of $20 \mathrm{MHz}$ varying the zenithal angle of the shower. We have simulated, for each zenithal angle, ten different showers and calculated the relative difference between the far-field and exact calculations. We have plotted the mean and standard deviation of these differences in Fig. 10, where we can conclude that at distances larger than $\sim 100 \mathrm{~m}$, the error for the inspected showers is less than $1 \%$ for the EW and north-south (NS) components. However, the error in the vertical component for a vertical shower (less than $20^{\circ}$ ) is quite large. For the depicted $0^{\circ}$ shower (Fig. 10, top left), even at $250 \mathrm{~m}$ from the shower core, the mean error lies around $\sim 30 \%$. For the inspected 0, 20, 40 degree showers, the error in the horizontal polarizations at distances larger than $100 \mathrm{~m}$ is of the order of $1 \%$ or less, but it does not decrease rapidly with the distance because of the shower particles that lie closer to the ground. Because of this, the error committed when calculating the field from a $60^{\circ}$ shower (Fig. 10, bottom right), which presents a smaller number of particles near the observer, is less than the error committed for vertical showers. We have checked that for showers having a zenith angle smaller than $40^{\circ}$ the error as a function of distance for $50 \mathrm{MHz}$ is similar to the error at $20 \mathrm{MHz}$. The above facts hint that for most current experiments measuring in the [20-80] $\mathrm{MHz}$ band and only horizontal polarizations, the far-field approximation rests valid at distances larger than $100 \mathrm{~m}$ from the shower core. If, however, an antenna lies at a smaller distance, or if the vertical component is being measured, one should consider using the exact formula presented in this work. For low-frequency experiments, the use of Eq. (21) is imperative, but remembering that Eq. (21) ignores the effect of the boundary, which is likely to give further corrections. 


\section{PROPERTIES OF THE SUDDEN DEATH PULSE}

\section{A. Simple model for the sudden death pulse}

We have obtained the SDP in our simulations as a pulse created by the sudden deceleration of the shower front upon its arrival at the ground. Because of the large extension of the shower front and its thickness that causes the particles to arrive at different times, the pulse is only coherent at low frequencies (Fig. 6). However, the Nishimura-KamataGreisen formula predicts that even for relative old showers with an age of $s=1.5$, around half of the particles are contained inside a distance to the shower axis less than the Molière radius, $\sim 100 \mathrm{~m}$. For a young shower with $s=1.2$, we have more particles inside. The Molière radius is comparable to the wavelengths between 1 and $5 \mathrm{MHz}$, that is, from 300 to $60 \mathrm{~m}$, which inspires us to postulate a simple model for the SDP, wherein all the particles travel parallel to the shower axis very close to each other and arrive at the shower core. The model implies that the bulk of the radiation is produced when the part of the shower with most particles suddenly decelerates, creating a maximum for the pulse of the form

$$
\begin{aligned}
\mathbf{E}_{\mathrm{SDP}, \max }(\mathbf{x}, t) & =-|e| \frac{N \Delta q}{4 \pi \epsilon R} \mathbf{v}_{\perp} \delta\left(t-\frac{n R}{c}\right) \\
& =-|e| \frac{N \Delta q}{4 \pi \epsilon R}(-\hat{\mathbf{R}} \times(\hat{\mathbf{R}} \times \mathbf{v})) \delta\left(t-\frac{n R}{c}\right),
\end{aligned}
$$

where we have applied the far-field approximation, and also that the core is located at the origin and the shower maximum arrives at the ground at $t=0 . N$ is the number of particles arriving at the ground, $\Delta q$ is the ratio of (negative) excess charge particles to the total number of particles, and $\mathbf{v}$ is the mean velocity of the shower particles along the shower axis. $\mathbf{v}_{\perp}=-\hat{\mathbf{R}} \times(\hat{\mathbf{R}} \times \mathbf{v})$ corresponds to its projection perpendicular to the line of sight that joins the shower core and the observer. Equation (34) gives already some insight on the expected maximum of the SDP. It implies the following:

(i) the arrival time of the maximum of the pulse is linear with the distance to the shower core, and the proportionality is precisely the inverse of the speed of light in the medium;

(ii) in the far field, we expect a maximum amplitude with a dependence of $1 / R$, akin to a radiation field, and

(iii) the polarization of this pulse should lie along the direction of the shower axis projected onto the direction perpendicular to the line of sight core observer, as a first approximation.

\section{B. Arrival time as a function of the distance to the shower core}

We have simulated a $1 \mathrm{EeV}$ proton-induced shower of $30^{\circ}$ of zenithal angle coming from the east and put several

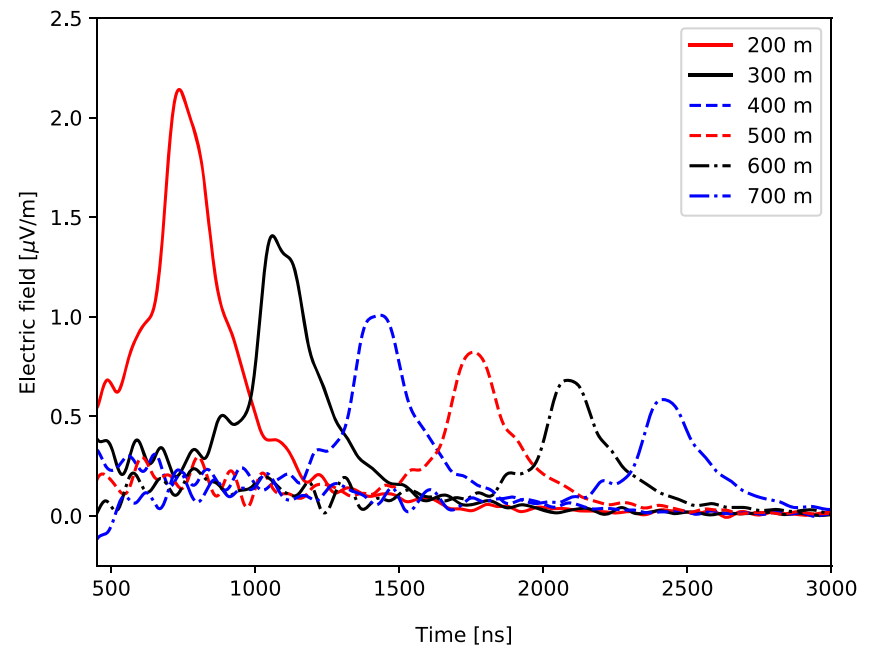

FIG. 11. SDP electric field (vertical component) as a function of time created by a $1 \mathrm{EeV}$ proton-induced shower with $30^{\circ}$ of zenithal angle and coming from the east $\left(\phi=0^{\circ}\right)$. Observers have been placed north of the shower core, at regular intervals of $100 \mathrm{~m}$. The dependence of the arrival time of the maxima with the distance, as well as the amplitude, is apparent. Traces have been filtered with a $10 \mathrm{MHz}$ sixth-order Butterworth low-pass filter.

observers north of the shower core. After filtering the traces with a $10 \mathrm{MHz}$ sixth-order Butterworth filter, we show the SDP electric field as a function of time for different distances to the shower core in Fig. 11, where it is apparent that the maxima present a displacement proportional to the distance. Moreover, the amplitude seems to decrease with the inverse of the distance. The traces in Fig. 11 present an asymmetry (the signal before the maximum is asymmetric with respect to the signal after the maximum) even at $700 \mathrm{~m}$ from the shower core, and since the SDP is created by the instantaneous deceleration of the shower particles at ground level, the asymmetry in the pulse reflects the asymmetry in the deceleration of ground particles and, therefore, of the shower disk projection on the ground.

The time arrival dependence is verified in Fig. 12, where we plot the arrival times (with arbitrary offsets for clarity) as a function of distance for showers similar to that in Fig. 11, but varying the energy. Each data set has a linear fit superimposed, whose slopes vary from 3.332 to $3.347 \mathrm{~ns} \mathrm{~m}^{-1}$, while the expected slope is $1 / c_{n}=n / c \approx 3.336 \mathrm{~ns} \mathrm{~m}^{-1}$. The simulated arrival time is, in fact, in good agreement with the simple model prediction.

\section{Amplitude as a function of the distance to the shower core}

The $1 / R$ dependence has been tested in a similar way, but in this case we have taken the mean of the SDP maximum amplitude for ten different simulated showers with the same geometry and energy as in Fig. 12. For each set of amplitudes $A_{i}$ we have calculated its mean $\langle A\rangle$ and 


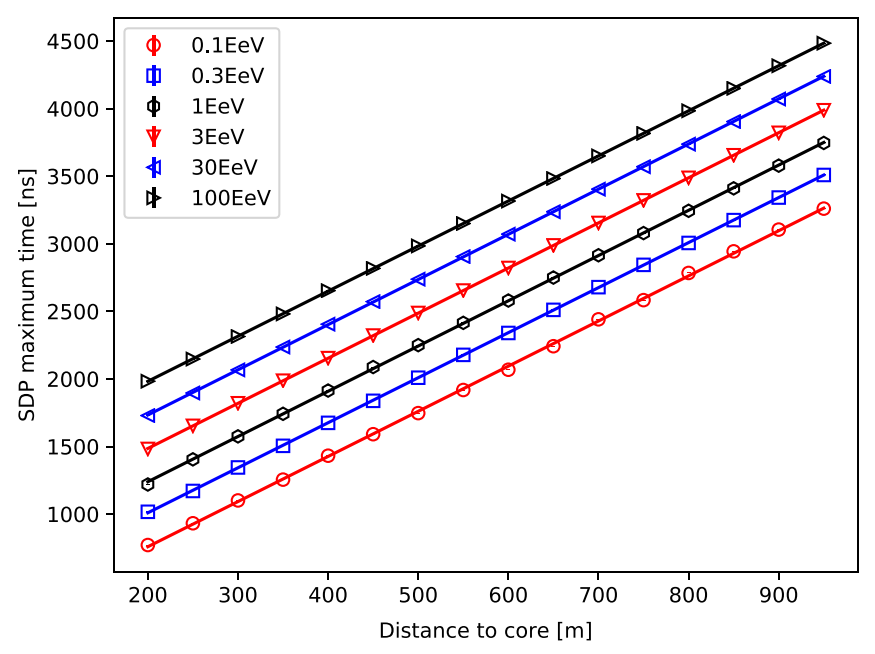

FIG. 12. Arrival time of the SDP maximum (vertical component) as a function of distance to the shower core for protoninduced showers of different energies (indicated on the legend) with $30^{\circ}$ of zenithal angle and coming from the east $\left(\phi=0^{\circ}\right)$. Observers have been placed north of the shower core. The superimposed lines correspond to a linear fit to the data, with a slope close to the inverse of the speed of light. Times have been offset for visual clarity. See text for details.

performed a fit of the form $\log _{10}\langle A\rangle=a+b \log _{10} R$, using as the uncertainty of $\log _{10}\langle A\rangle$ an estimation of the uncertainty of the mean of the logarithm of the amplitude, that is,

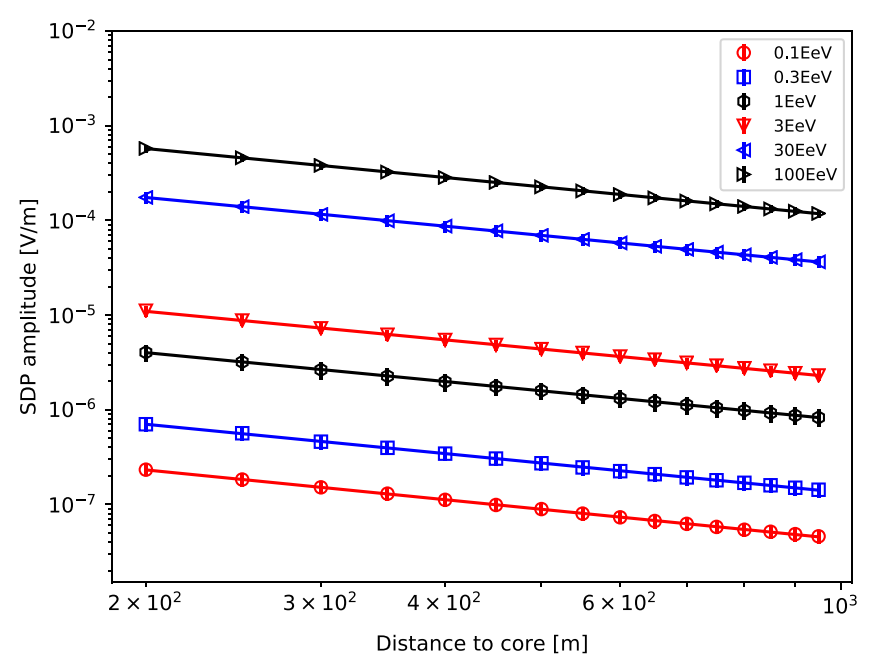

FIG. 13. Mean amplitude of the SDP maximum (vertical polarization) as a function of distance to the shower core, averaged over ten proton-induced showers of different energies (indicated on the legend) with $30^{\circ}$ of zenithal angle and coming from the east $\left(\phi=0^{\circ}\right)$. Observers have been placed north of the shower core. The superimposed lines correspond to a linear fit to the logarithm of the amplitude versus the logarithm of the distance, with the uncertainties given by Eq. (35) and represented by the small vertical lines at the center of each marker. The data are compatible with a $1 / R$ dependence. See text for details.

$$
\sigma=\sqrt{\frac{1}{n(n-1)}\left[\left\langle\left(\log _{10} A\right)^{2}\right\rangle-\left\langle\log _{10} A\right\rangle^{2}\right]} .
$$

We have plotted the results in Fig. 13. The slope of the fit varies from -1.04 to 1.00 , being compatible with -1 (pure radiation field) at $1 \sigma$ below $1 \mathrm{EeV}$ and $2 \sigma$ above. In any case, the data are very close to what one would expect from a pure radiation field, agreeing with our simple model.

\section{Amplitude as a function of the primary energy}

Another feature of the SDP that can be drawn from Eq. (34) is that we expect an amplitude proportional to the number of particles that reach the ground. This implies that, statistically, the higher the energy, the larger the amplitude of the SDP, although we do not expect a linear dependence, since the number of particles that arrive at the ground is not linear with the energy due to the variation of the shower maximum depth with the primary energy. We can, nonetheless, plot the expected SDP amplitude as a function of the primary energy for several distances to the shower core, as in Fig. 14, and fit the logarithm of the SDP amplitude. The values for the slope are not compatible with 1 , as expected, but the $\chi^{2} /$ ndof of the fits are, from smaller to larger distance, $0.75,1.07,1.57$, and 0.53 , while the $90 \%$ C.L. for a $\chi^{2}$ with 4 degrees of freedom is $\chi^{2} /$ ndof $_{0.9}=1.99475$, meaning that the amplitude can be related to a power of the primary energy for these

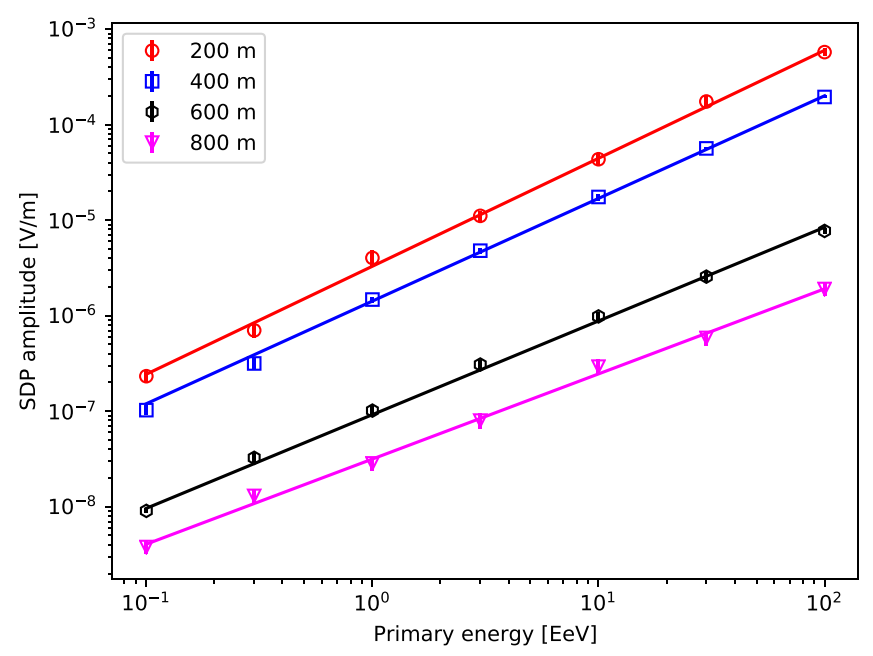

FIG. 14. Mean amplitude of the SDP maximum (vertical polarization) as a function of the primary energy, averaged over ten proton-induced showers with $30^{\circ}$ of zenithal angle and coming from the east $\left(\phi=0^{\circ}\right)$. Observers have been placed north of the shower core (see legend). The superimposed lines correspond to a linear fit to the logarithm of the amplitude versus the logarithm of the primary energy, with the uncertainty of the mean represented by the small vertical lines at the center of each marker. The simulated data are compatible with a power law of the type $A_{\mathrm{SDP}}=a E_{\text {primary }}^{b}$. See text for details. 
particular cases. $200 \mathrm{~m}$ away from the shower core, we find $\propto E^{1.13 \pm 0.02}$ and $800 \mathrm{~m}$ away we find $\propto E^{0.89 \pm 0.02}$. However, according to our model, both should present the same exponent, but this discrepancy is understandable because our model does not take into account the three-dimensional shape of the shower, which is crucial for a fine calculation of the electric field. In particular, the shape of the distribution of ground particles changes in a nonlinear way with the energy of the primary particle, and this distribution of ground particles is the one that determines the characteristics of the SDP as a function of the observer position.

\section{E. Spectrum}

In Fig. 15 (top) we find an example of the spectrum for the principal pulse and the SDP created by a $1 \mathrm{EeV}$ and $30^{\circ}$
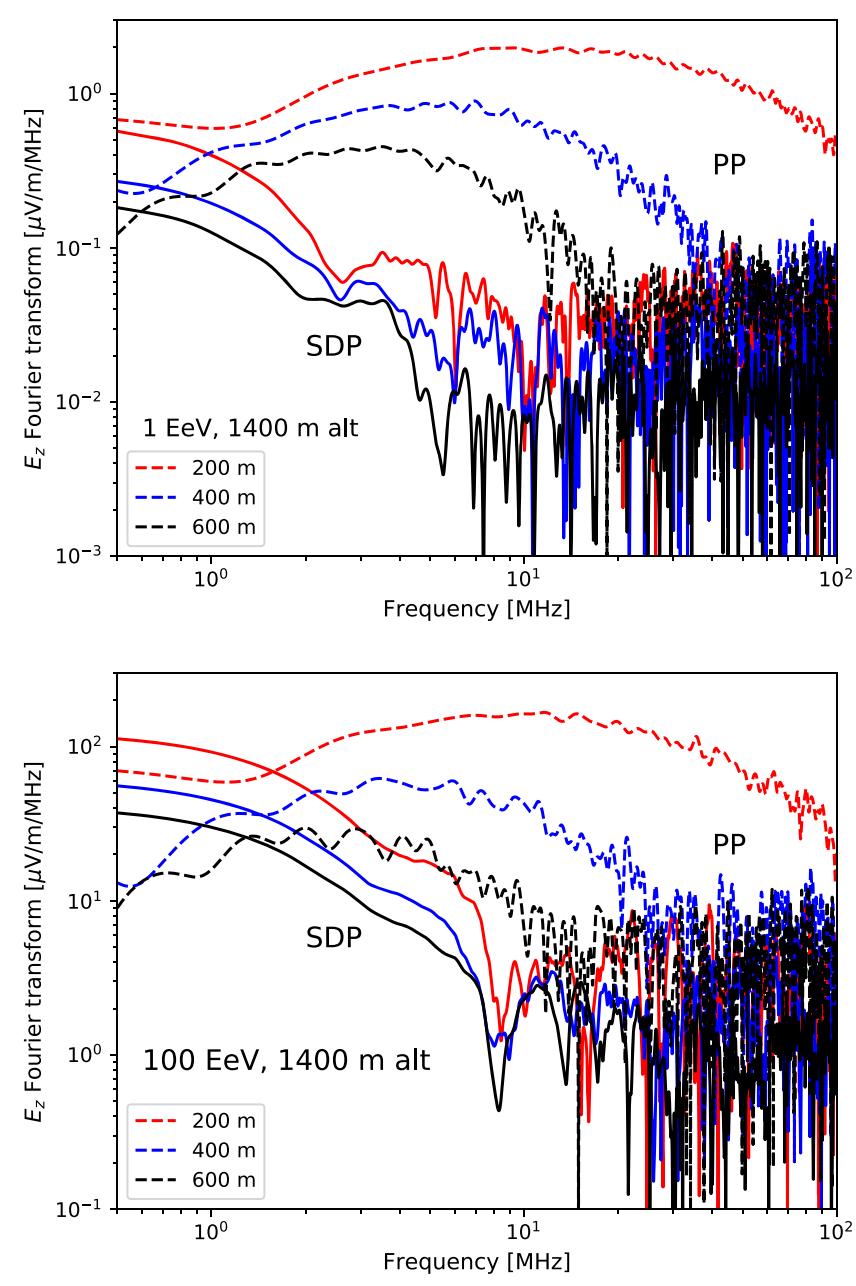

FIG. 15. Top: Amplitude of the vertical component of the Fourier transform of the principal pulse (dashed lines) and the SDP (solid lines) as a function of frequency created by a $1 \mathrm{EeV}$ proton-induced shower with $30^{\circ}$ of zenithal angle and coming from the east $\left(\phi=0^{\circ}\right)$. We have placed observers 200, 400, and $600 \mathrm{~m}$ north of the shower core, located on the ground at $1400 \mathrm{~m}$ of altitude. Each pulse presents a different frequency dependence. Bottom: Same as top, but for $100 \mathrm{EeV}$ of primary energy. proton-induced shower. The principal pulse presents the well-understood shape of growing electric field up to a certain frequency upon which the emission ceases to be coherent, falls off, and then stabilizes at high frequencies as an incoherent spectrum. The SDP, on the contrary, is much larger at low frequencies and becomes incoherent at $\sim 10 \mathrm{MHz}$, indicating that the SDP should be observable only at frequencies below $10 \mathrm{MHz}$. Increasing the energy increases the principal pulse, but also the SDP, each one in a different way. In fact, for a $100 \mathrm{EeV}$ shower (Fig. 15, bottom), the SDP at low frequencies $(<2 \mathrm{MHz})$ is larger than the principal pulse.

\section{F. Amplitude vs number of ground particles}

According to Eq. (34), we expect the maximum amplitude of the SDP to be proportional to the excess of negatively charged particles arriving at the ground. If we choose the vector $\hat{\mathbf{R}}$ to be perpendicular to $\mathbf{v}$, the total maximum amplitude of the pulse in our simple model is equal to

$$
\left|\mathbf{E}_{\mathrm{SDP}, \max }(\mathbf{x}, t)\right|=\frac{e N \Delta q}{4 \pi \epsilon R} \delta\left(t-\frac{n R}{c}\right) \propto N,
$$

which is effectively proportional to the number of ground particles. Let us place an observer $200 \mathrm{~m}$ north of the shower core and let us simulate the electric fields from showers coming from the east at zenith angles from 0 to $80^{\circ}$ and primary energies ranging from 0.1 to $100 \mathrm{EeV}$. We show the results in Fig. 16 for a ground at $180 \mathrm{~m}$ of altitude, similar to that which the EXTASIS experiment rests on. We show in Fig. 16, top left, the number of electrons and positrons arriving at the ground as a function of the primary energy and shower zenith angle, while at the top right part of the figure we find the same plot for the maximum total amplitude of the SDP. Both figures are similar, indicating a correlation between number of particles and pulse amplitude. We show in Fig. 16, bottom left, the same plot for the vertical polarization and at the bottom right the EW polarization.

Let us increase the altitude to $1400 \mathrm{~m}$. An increase in the number of particles and the SDP amplitude is expected, which is precisely what we find in Fig. 17. Supposing a detection threshold of $5 \mu \mathrm{V} / \mathrm{m}$, detectable showers at $180 \mathrm{~m}$ of altitude should have an energy larger than $1 \mathrm{EeV}$ (see the region at the right of the $5 \mu \mathrm{V} / \mathrm{m}$ contour line, Fig. 16, top right). For the EXTASIS site and the projected livetime of $\sim 2,3$ years we do not expect showers having more energy than $10 \mathrm{EeV}$, so we aim to detect the SDP coming from showers with energies $>1 \mathrm{EeV}$ and having a zenith angle $<30^{\circ}$. The more vertical the shower, the easier it should be to detect. For a site at $1400 \mathrm{~m}$ of altitude (Fig. 17, right), the range of detectability is larger, and it is even larger for a $2650 \mathrm{~m}$ altitude, similar to the one at the GRAND site [48]. 

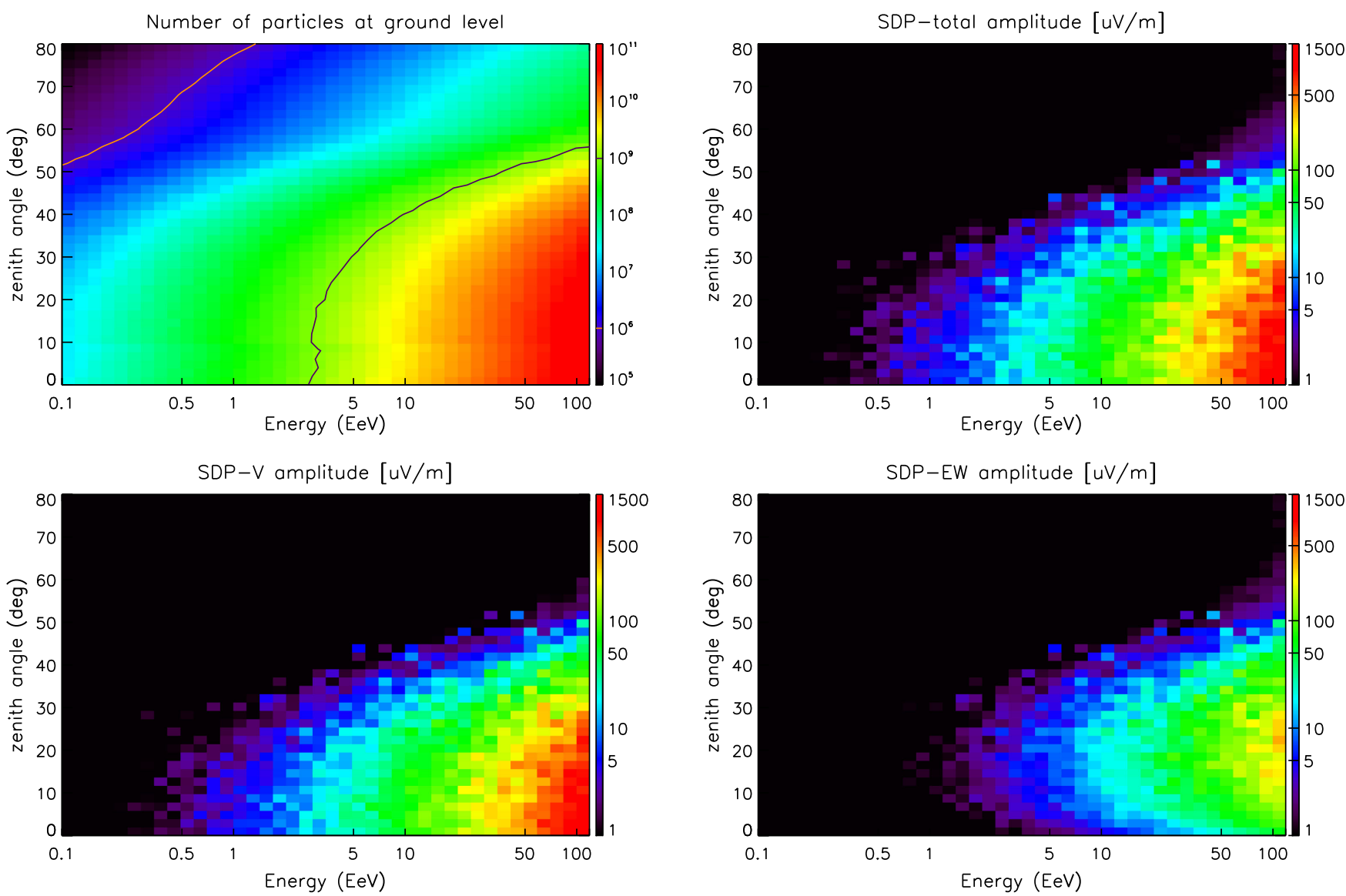

FIG. 16. Top left: Two-dimensional color plot of the number of electrons and positrons arriving at the ground (180 $\mathrm{m}$ of altitude) as a function of the primary proton energy and shower zenith angle. Each bin contains the mean number of particles averaged over 100 showers. Top right: Two-dimensional color plot of the total SDP maximum amplitude (three polarizations) as a function of the primary proton energy and shower zenith angle. Each bin contains the mean amplitude averaged over five showers. Bottom left: Same as top right, for the vertical polarization. Bottom right: Same as top right, for the EW polarization.
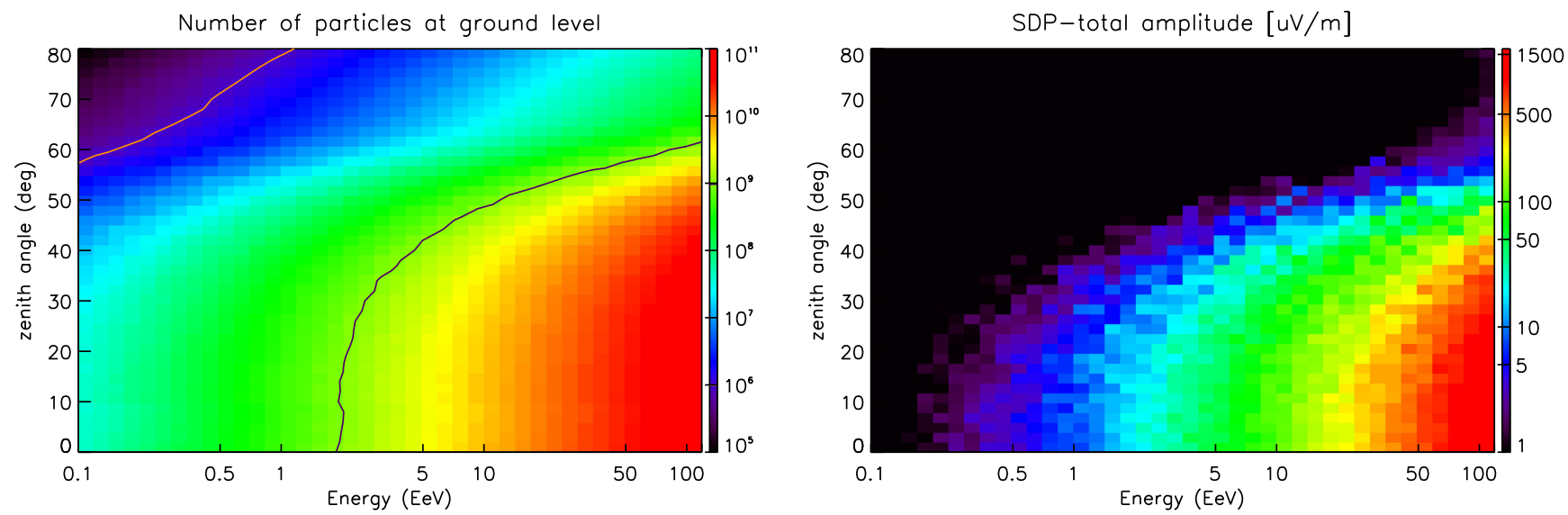

FIG. 17. Same as Fig. 16, top, but with a ground altitude equal to $1400 \mathrm{~m}$.

\section{SUMMARY AND CONCLUSIONS}

We have presented a time-domain equation of the electric field produced by a particle track, defined as a charged particle that accelerates instantaneously, moves at a constant speed, and instantaneously decelerates. Special emphasis has been put on the conservation of the total charge so as to ensure that the equation is a valid solution of Maxwell's equations and therefore gives the correct physical field. Explicitly conserving the charge results in the appearance of 
two impulse fields coming from the first and last points of the trajectory of the particle track and that needs to be added to the total field. We have checked that our complete equation reduces in the far-field limit $(k R \gg 1)$ to the ZHS formula, which has been proven to be a correct expression for the farfield emission of a particle track.

We have also solved the exact electric field for a track in frequency (Fourier) domain, starting from the same physical problem (same set of charge densities and current densities) but solving Maxwell's equations in frequency domain. We have shown that the result is analytically equivalent to the Fourier transform of the time-domain electric field. This is a check of the correctness of the formula as well as a confirmation that working with the time-domain formula and then transforming to frequency domain is mathematically sound, since both equations are Fourier transforms of each other. Limitations in validity and precision come from the numerical step chosen for the evaluation of the time-domain field and from the discrete Fourier transform employed. Moreover, the frequencydomain version of the electric field for a track is numerically equivalent to another frequency-domain formula [37] obtained with the same hypotheses but with a different way of calculating the scalar potential, in what constitutes another check for our expression.

We have implemented the formula in the SELFAS Monte Carlo code so as to know the emission it predicts for EAS. In doing so, we neglected the static fields created after the particle flight since this physical situation is not going to be given in a real EAS, for the atmosphere will tend to regain electrostatic equilibrium as soon as possible. After the implementation, the results of our simulations show that for a frequency larger than $20 \mathrm{MHz}$ and horizontal polarizations (the case for experiments like AERA, CODALEMA, LOFAR or Tunka-Rex), the farfield approximation can be used with an error of the order of $\sim 1 \%$ or lower at distances greater than $100 \mathrm{~m}$ from the shower core. However, for smaller distances to the shower core one may consider using the exact formula for a particle track. In the case of measuring at a frequency lower than $20 \mathrm{MHz}$ or measuring the vertical component of an EAS, as is the case for the EXTASIS experiment, the use of the exact formula [Eq. (21)] is unavoidable if one is to know the accurate electric field. For experiments conducted in dense media, such as ARA, we expect the far-field approximation to be valid almost always due to the much smaller size of the shower, as shown in [37].

We have shown a low-frequency impulse field created by the coherent deceleration of the shower front upon its arrival to the ground, which we have called the SDP. This pulse vanishes for frequencies above $20 \mathrm{MHz}$, which means that such a field could be detectable with dedicated lowfrequency experiments like EXTASIS. In a first-order approximation, we have calculated only the direct emission of the decelerating shower front, ignoring the induced surface wave.
We have studied the properties of the direct emission of the SDP. The arrival time of the shower maximum is linear with the distance from the observer to the shower core, and the proportionality constant is compatible with the inverse of the speed of light. The amplitude of the SDP decays with the inverse of the distance to the shower core as well, which means that the SDP behaves similarly to a radiation field. These results can be explained using a simple model consisting in a long track containing all the excess of charged particles from the shower that arrive at the ground and calculating the field created in the instant the track suddenly decelerates.

The amplitude of the SDP has been found to be a monotonously increasing function of the primary energy, and, in particular, it is proportional to the number of particles arriving at the ground. The vertical polarization is favored for vertical showers while the horizontal polarizations are more present the more inclined the shower is, as suggested by the simple model proposed here.

One important caveat to keep in mind is that while the exact field of a particle track is needed if we want to calculate the near-field emission of an EAS, one has to know the response of the measuring antenna to an incoming near-field emission to elucidate the final antenna voltage. In this work we have been concerned with obtaining a physical electric field, but the link to the antenna voltage, which is what is measured eventually, lies outside the scope of this paper.

Finally, while the SDP explains some of the properties of the pulses measured by the low-frequency experiments in the past, the SDP amplitudes obtained in the present work are not large enough to explain all the experimental data. A study on the influence of the surface wave may help us to solve this discrepancy. This work is underway.

\section{ACKNOWLEDGMENTS}

We thank the Région Pays de la Loire for its financial support of the Groupe Astro of Subatech and in particular, for its contribution to the EXTASIS experiment.

\section{APPENDIX A: OBTENTION OF THE ZHS FORMULA}

Let us begin by dropping all the terms with a $1 / R^{2}$ dependence in Eq. (21),

$$
\begin{aligned}
\mathbf{E}(\mathbf{x}, t)= & \frac{q}{4 \pi \epsilon}\left\{-\frac{\hat{\mathbf{R}}_{1}}{c_{n} R_{1}} \delta\left(t-t_{1}-R_{1} / c_{n}\right)\right. \\
& +\frac{\hat{\mathbf{R}}_{2}}{c_{n} R_{2}} \delta\left(t-t_{2}-R_{2} / c_{n}\right) \\
& +\frac{1}{c_{n} R} \frac{\partial}{\partial t}\left[\frac{\hat{\mathbf{R}}}{\kappa}\left(\Pi\left(t^{\prime}, t_{1}, t_{2}\right)\right)\right]_{\mathrm{ret}} \\
& \left.-\frac{\mathbf{v}}{c_{n}^{2} R} \frac{\partial}{\partial t}\left[\frac{1}{\kappa}\left(\Pi\left(t^{\prime}, t_{1}, t_{2}\right)\right)\right]_{\mathrm{ret}}\right\} .
\end{aligned}
$$


Let us suppose now that the unit vector $\hat{R}$ and $\kappa=|1-n \beta \cos \theta|$ do not change during the track flight, which is equivalent to having the observation angle $\theta$ limited to a narrow range or having the track far away from the observers. Let us take $R \approx R_{0}$, with $R_{0}$ being one of the points in the track, for instance, the center point. Partial time derivatives are then 0 except when the particle accelerates and decelerates,

$$
\begin{aligned}
\mathbf{E}(\mathbf{x}, t)= & \frac{q}{4 \pi \epsilon}\left\{-\frac{\hat{\mathbf{R}}_{0}}{c_{n} R_{0}} \delta\left(t-t_{1}-R_{1} / c_{n}\right)+\frac{\hat{\mathbf{R}}_{0}}{c_{n} R_{0}} \delta\left(t-t_{2}-R_{2} / c_{n}\right)\right. \\
& \pm \frac{1}{c_{n}} \frac{\hat{\mathbf{R}}_{0}}{\kappa R_{0}} \delta\left(t-t_{1}-R_{1} / c_{n}\right) \mp \frac{\mathbf{v}}{c_{n}^{2} \kappa R_{0}} \delta\left(t-t_{1}-R_{1} / c_{n}\right) \\
& \left.\mp \frac{1}{c_{n}} \frac{\hat{\mathbf{R}}_{0}}{\kappa R_{0}} \delta\left(t-t_{2}-R_{2} / c_{n}\right) \pm \frac{\mathbf{v}}{c_{n}^{2} \kappa R_{0}} \delta\left(t-t_{2}-R_{2} / c_{n}\right)\right\},
\end{aligned}
$$

where from the \pm and $\mp$ symbols, we take the upper one if $\theta>\theta_{C}$ and the lower one if otherwise. Since we are in the far field, we can use the Fraunhofer approximation for the distance: choosing a point $\mathbf{x}_{0}$ inside the track, and supposing the particle is at $\mathbf{x}_{0}$ when $t^{\prime}=t_{0}=0$,

$$
R_{i}=\left|\mathbf{x}-\mathbf{x}_{0}+\mathbf{v} t^{\prime}\right| \approx R_{0}-\mathbf{v} \cdot \hat{\mathbf{R}}_{0} t_{i}=R_{0}-\cos \theta v t_{i} .
$$

Plugging Eq. (A3) into Eq. (A2) and renaming $R_{0} \rightarrow R$,

$$
\begin{aligned}
\mathbf{E}(\mathbf{x}, t)= & \frac{q}{4 \pi \epsilon}\left\{-\frac{\hat{\mathbf{R}}}{c_{n} R} \delta\left(t-\frac{n R}{c}-(1-n \beta \cos \theta) t_{1}\right)+\frac{\hat{\mathbf{R}}}{c_{n} R} \delta\left(t-\frac{n R}{c}-(1-n \beta \cos \theta) t_{2}\right)\right. \\
& \pm \frac{1}{c_{n}} \frac{\hat{\mathbf{R}}}{\kappa R} \delta\left(t-\frac{n R}{c}-(1-n \beta \cos \theta) t_{1}\right) \mp \frac{\mathbf{v}}{c_{n}^{2} \kappa R} \delta\left(t-\frac{n R}{c}-(1-n \beta \cos \theta) t_{1}\right) \\
& \left.\mp \frac{1}{c_{n}} \frac{\hat{\mathbf{R}}}{\kappa R} \delta\left(t-\frac{n R}{c}-(1-n \beta \cos \theta) t_{2}\right) \pm \frac{\mathbf{v}}{c_{n}^{2} \kappa R} \delta\left(\left(t-\frac{n R}{c}-(1-n \beta \cos \theta) t_{2}\right)\right)\right\} .
\end{aligned}
$$

We now make use of the following identity:

$$
\hat{\mathbf{R}}-\frac{\hat{R}}{\kappa}+\frac{\mathbf{v}}{\kappa c_{n}}= \pm \frac{1}{\kappa}\left[\frac{\mathbf{v}}{c_{n}}-n \beta \hat{\mathbf{R}} \cos \theta\right]= \pm \frac{1}{\kappa c_{n}}[\mathbf{v}(\hat{\mathbf{R}} \cdot \hat{\mathbf{R}})-\hat{\mathbf{R}}(\mathbf{v} \cdot \hat{\mathbf{R}})]= \pm \frac{1}{\kappa c_{n}}[-\hat{\mathbf{R}} \times(\hat{\mathbf{R}} \times \mathbf{v})]= \pm \frac{\mathbf{v}_{\perp}}{\kappa c_{n}},
$$

where we take the plus sign if $\theta>\theta_{C}$ and the minus sign if otherwise. $\mathbf{v}_{\perp}$ is, by definition, the projection of the particle velocity perpendicular to the line joining the particle and the observer, also called the line of sight. With Eq. (A5), using a positron charge defined as $q=e>0$ and using $1 /\left(\epsilon c_{n}^{2}\right)=\mu_{r} /\left(\epsilon_{0} c^{2}\right)$, we arrive at the same equation as in [35],

$$
\mathbf{E}_{\mathrm{ZHS}}(\mathbf{x}, t)=-\frac{e \mu_{r}}{4 \pi \epsilon_{0} c^{2} R} \mathbf{v}_{\perp} \frac{\delta\left(t-\frac{n R}{c}-(1-n \beta \cos \theta) t_{1}\right)-\delta\left(t-\frac{n R}{c}-(1-n \beta \cos \theta) t_{2}\right)}{1-n \beta \cos \theta} .
$$

Note that the \pm and the $\mp$ have been substituted by the sign of the denominator that is positive outside the Cherenkov cone and negative inside.

\section{APPENDIX B: TRANSFORMATION OF EQ. (21) TO FREQUENCY DOMAIN}

Equation (21) can be transformed to frequency domain. Defining the Fourier transform of a function $f(t)$ as

$$
F(\omega)=\int_{-\infty}^{+\infty} \mathrm{d} t e^{i \omega t} f(t)
$$

and knowing the Fourier transform for a step function,

$$
\int_{-\infty}^{+\infty} \mathrm{d} t e^{i \omega t} \Theta\left(t-t_{0}\right)=e^{i \omega t_{0}}\left[\frac{i}{\omega}+\pi \delta(\omega)\right]
$$

we find that 


$$
-\frac{q}{4 \pi \epsilon} \int_{-\infty}^{+\infty} \mathrm{d} t \frac{\hat{\mathbf{R}}_{1}}{R_{1}^{2}} \Theta\left(t-t_{1}-R_{1} / c_{n}\right)=-\frac{q}{4 \pi \epsilon} \frac{\hat{\mathbf{R}}_{1}}{R_{1}^{2}} e^{i \omega t_{1}} e^{i k R_{1}}\left[\frac{i}{\omega}+\pi \delta(\omega)\right],
$$

with $k=\omega / c_{n}$ and

$$
-\frac{q}{4 \pi \epsilon c_{n}} \int \mathrm{d} t e^{i \omega t} \frac{\hat{\mathbf{R}}_{1}}{R_{1}^{2}} \delta\left(t-t_{1}-R_{1} / c_{n}\right)=-\frac{q}{4 \pi \epsilon c_{n}} e^{i \omega t_{1}} e^{i k R_{1}} \frac{\hat{\mathbf{R}}_{1}}{R_{1}^{2}}
$$

The two terms for the deceleration can be written following the same steps. Taking the first term of the second line in Eq. (21) and transforming it to frequency, supposing that $\theta>\theta_{C}$,

$$
\begin{aligned}
\frac{q}{4 \pi \epsilon} \int_{-\infty}^{+\infty} \mathrm{d} t e^{i \omega t}\left[\frac{\hat{\mathbf{R}}}{\kappa R^{2}}\left(\Theta\left(t^{\prime}-t_{1}\right)-\Theta\left(t^{\prime}-t_{2}\right)\right)\right]_{\mathrm{ret}} & =\frac{q}{4 \pi \epsilon} \int_{t_{1}+R_{1} / c_{n}}^{t_{2}+R_{2} / c_{n}} \mathrm{~d} t e^{i \omega t}\left[\frac{\hat{\mathbf{R}}}{\kappa R^{2}}\right]_{\mathrm{ret}} \\
& =\frac{q}{4 \pi \epsilon} \int_{t_{1}}^{t_{2}} \mathrm{~d} t^{\prime} e^{i \omega t^{\prime}} e^{i k R\left(t^{\prime}\right)} \frac{\hat{\mathbf{R}}}{\kappa R^{2}} \frac{\mathrm{d} t}{\mathrm{~d} t^{\prime}}=\frac{q}{4 \pi \epsilon} \int_{t_{1}}^{t_{2}} \mathrm{~d} t^{\prime} e^{i \omega t^{\prime}} e^{i k R\left(t^{\prime}\right)} \frac{\hat{\mathbf{R}}}{R^{2}},
\end{aligned}
$$

where we have made the change of variable $t \rightarrow t^{\prime}$. Taking a geometry in which $\theta<\theta_{C}$ instead yields

$$
\frac{q}{4 \pi \epsilon} \int_{t_{2}+R_{2} / c_{n}}^{t_{1}+R_{1} / c_{n}} \mathrm{~d} t e^{i \omega t}\left[\frac{\hat{\mathbf{R}}}{\kappa R^{2}}\right]_{\text {ret }}=\frac{q}{4 \pi \epsilon} \int_{t_{2}}^{t_{1}} \mathrm{~d} t^{\prime} e^{i \omega t^{\prime}} e^{i k R\left(t^{\prime}\right)} \frac{\hat{\mathbf{R}}}{\kappa R^{2}}(-\kappa)=\frac{q}{4 \pi \epsilon} \int_{t_{1}}^{t_{2}} \mathrm{~d} t^{\prime} e^{i \omega t^{\prime}} e^{i k R\left(t^{\prime}\right)} \frac{\hat{\mathbf{R}}}{R^{2}},
$$

which is exactly the same result as Eq. (B5). In fact, if we suppose that for a point along the track, $\theta=\theta_{C}$, and we also suppose that the arrival time of the shockwave to the observer is $t_{C, \mathrm{obs}}=t_{C}+R_{C} / c_{n}$, the transform is

$$
\begin{aligned}
& \frac{q}{4 \pi \epsilon}\left[\int_{t_{C, \mathrm{obs}}}^{t_{1}+R_{1} / c_{n}} \mathrm{~d} t e^{i \omega t}\left[\frac{\hat{\mathbf{R}}}{\kappa R^{2}}\right]_{\mathrm{ret}}+\int_{t_{C, \mathrm{obs}}}^{t_{1}+R_{1} / c_{n}} \mathrm{~d} t e^{i \omega t}\left[\frac{\hat{\mathbf{R}}}{\kappa R^{2}}\right]_{\mathrm{ret}}\right] \\
& =\frac{q}{4 \pi \epsilon}\left[\int_{t_{1}}^{t_{C}} \mathrm{~d} t^{\prime} e^{i \omega t^{\prime}} e^{i k R\left(t^{\prime}\right)} \frac{\hat{\mathbf{R}}}{R^{2}}+\int_{t_{C}}^{t_{2}} \mathrm{~d} t^{\prime} e^{i \omega t^{\prime}} e^{i k R\left(t^{\prime}\right)} \frac{\hat{\mathbf{R}}}{R^{2}}\right]=\frac{q}{4 \pi \epsilon} \int_{t_{1}}^{t_{2}} \mathrm{~d} t^{\prime} e^{i \omega t^{\prime}} e^{i k R\left(t^{\prime}\right)} \frac{\hat{\mathbf{R}}}{R^{2}},
\end{aligned}
$$

exactly the same as Eqs. (B5) and (B6). We can transform directly the rest of the terms of the second line in Eq. (21) using the derivative property of the Fourier transform,

$$
\int \mathrm{d} t e^{i \omega t} \frac{\partial f(\mathbf{x}, t)}{\partial t}=-i \omega \int \mathrm{d} t e^{i \omega t} f(\mathbf{x}, t),
$$

since for our function, $\lim _{t \rightarrow \pm \infty} f(\mathbf{x}, t)=0$. Together with Eq. (B7) we write the following exact field for a track in frequency:

$\mathbf{E}(\mathbf{x}, \omega)=\frac{q}{4 \pi \epsilon}\left\{-\frac{\hat{\mathbf{R}}_{1}}{R_{1}} e^{i \omega t_{1}} e^{i k R_{1}}\left[\frac{i}{\omega R_{1}}+\frac{1}{c_{n}}\right]+\int_{t_{1}}^{t_{2}} \mathrm{~d} t^{\prime} e^{i \omega t^{\prime}} e^{i k R}\left[\frac{\hat{\mathbf{R}}}{R^{2}}-\frac{i \omega}{c_{n}} \frac{\hat{\mathbf{R}}}{R}+\frac{i \omega}{c_{n}^{2}} \frac{\mathbf{v}}{R}\right]+\frac{\hat{\mathbf{R}}_{2}}{R_{2}} e^{i \omega t_{2}} e^{i k R_{2}}\left[\frac{i}{\omega R_{2}}+\frac{1}{c_{n}}\right]\right\}$.

We have dropped the terms involving $\delta(\omega)$, since we are not interested in the contribution at zero frequency.

\section{APPENDIX C: DERIVATION OF THE ELECTRIC FIELD FOR A PARTICLE TRACK IN FREQUENCY DOMAIN}

Equation (27) can be derived directly in frequency domain. Let us assume, without loss of generality, a particle at rest that begins to move at a time $t_{1}$ and at the vertical coordinate $z=z_{1}$ with a velocity $\mathbf{v}=v \hat{z}$. The potentials in frequency domain can be written as

$$
\begin{aligned}
& \Phi(\mathbf{x}, \omega)=\frac{1}{4 \pi \epsilon} \int \mathrm{d}^{3} x^{\prime} \frac{e^{i k\left|\mathbf{x}-\mathbf{x}^{\prime}\right|}}{\left|\mathbf{x}-\mathbf{x}^{\prime}\right|} \rho\left(\mathbf{x}^{\prime}, \omega\right), \\
& \mathbf{A}(\mathbf{x}, \omega)=\frac{\mu_{0}}{4 \pi} \int \mathrm{d}^{3} x^{\prime} \frac{e^{i k\left|\mathbf{x}-\mathbf{x}^{\prime}\right|}}{\left|\mathbf{x}-\mathbf{x}^{\prime}\right|} \mathbf{J}\left(\mathbf{x}^{\prime}, \omega\right) .
\end{aligned}
$$

It should be noted that the changes $\epsilon \rightarrow \epsilon(\omega)$ and $\mu_{0} \rightarrow \mu(\omega)$ are always possible, so we can always generalize our final result for a frequency-dependent dielectric and magnetic medium. The charge density and the current density are steadily written as 


$$
\begin{gathered}
\rho\left(\mathbf{x}^{\prime}, \omega\right)=q \int_{-\infty}^{\infty} \mathrm{d} t^{\prime} e^{i \omega t^{\prime}}\left[\delta^{3}\left(\mathbf{x}^{\prime}-\mathbf{x}_{1}\right) \Theta\left(t^{\prime}-t_{1}\right)+\delta^{3}\left(\mathbf{x}^{\prime}-\mathbf{x}_{p}\left(t^{\prime}\right)\right)\left(\Theta\left(t^{\prime}-t_{1}\right)-\Theta\left(t^{\prime}-t_{2}\right)\right)+\delta^{3}\left(\mathbf{x}^{\prime}-\mathbf{x}_{2}\right) \Theta\left(t^{\prime}-t_{2}\right)\right], \\
\mathbf{J}\left(\mathbf{x}^{\prime}, \omega\right)=q v \hat{z} \int_{-\infty}^{\infty} \mathrm{d} t^{\prime} e^{i \omega t^{\prime}} \delta^{3}\left(\mathbf{x}^{\prime}-\mathbf{x}_{p}\left(t^{\prime}\right)\right)\left(\Theta\left(t^{\prime}-t_{1}\right)-\Theta\left(t^{\prime}-t_{2}\right)\right),
\end{gathered}
$$

with $\mathbf{x}_{p}\left(t^{\prime}\right)=\mathbf{x}_{1}+\mathbf{v}\left(t^{\prime}-t_{1}\right)=z_{1} \hat{z}+v \hat{z}\left(t^{\prime}-t_{1}\right)$. Using Eq. (C3), we split Eq. (C1) into three terms,

$$
\Phi(\mathbf{x}, \omega)=\frac{q}{4 \pi \epsilon}\left[-\int_{t_{1}}^{\infty} \mathrm{d} t^{\prime} \frac{e^{i k R_{1}}}{R_{1}} e^{i \omega t^{\prime}}+\int_{t_{2}}^{\infty} \mathrm{d} t^{\prime} \frac{e^{i k R_{2}}}{R_{2}} e^{i \omega t^{\prime}}+\int_{t_{1}}^{t_{2}} \mathrm{~d} t^{\prime} \frac{e^{i k R}}{R} e^{i \omega t^{\prime}}\right],
$$

since $R=\left|\mathbf{x}_{p}\left(t^{\prime}\right)-\mathbf{x}\right|$ after integrating the three-dimensional Dirac deltas. Equivalently,

$$
\Phi(\mathbf{x}, \omega)=\Phi_{1}+\Phi_{2}+\Phi_{p} .
$$

Equation $(\mathrm{C} 2)$ can be written as

$$
\mathbf{A}(\mathbf{x}, \omega)=\frac{\mu_{0}}{4 \pi} \int_{t_{1}}^{t_{2}} \mathrm{~d} t^{\prime} \frac{e^{i k R}}{R} e^{i \omega t^{\prime}} \mathbf{v} .
$$

Because of the cylindrical symmetry of our problem, we can calculate the radial component of the field using $\Phi$ alone.

$$
E_{\rho}=-\frac{\partial}{\partial \rho}(\Phi)=-\frac{\partial}{\partial \rho}\left(\Phi_{1}+\Phi_{2}+\Phi_{p}\right)
$$

using that

$$
\frac{\partial}{\partial x_{i}}\left(\frac{e^{i k\left|\mathbf{x}-\mathbf{x}^{\prime}\right|}}{\left|\mathbf{x}-\mathbf{x}^{\prime}\right|}\right)=\frac{e^{i k\left|\mathbf{x}-\mathbf{x}^{\prime}\right|}}{\left|\mathbf{x}-\mathbf{x}^{\prime}\right|}\left[i k-\frac{1}{\left|\mathbf{x}-\mathbf{x}^{\prime}\right|}\right] \frac{\hat{x}_{i} \cdot\left(\mathbf{x}-\mathbf{x}^{\prime}\right)}{\left|\mathbf{x}-\mathbf{x}^{\prime}\right|},
$$

where $x_{i}=x, y, z$ but it can also be $\rho$, the radial coordinate. Let us write

$$
-\frac{\partial}{\partial \rho} \Phi_{p}=\frac{q}{4 \pi \epsilon} \int_{t_{1}}^{t_{2}} \mathrm{~d} t^{\prime} \frac{e^{i k R}}{R} e^{i \omega t^{\prime}}\left[\frac{1}{R}-\frac{i \omega}{c_{n}}\right] \hat{\mathbf{R}} \cdot \hat{\rho} .
$$

Using the transform of the step function, we find the derivatives of $\Phi_{1}$ and $\Phi_{2}$.

$$
\begin{gathered}
-\frac{\partial}{\partial \rho} \Phi_{1}=-\frac{q}{4 \pi \epsilon} \int_{t_{1}}^{\infty} \mathrm{d} t^{\prime} \frac{e^{i k R_{1}}}{R_{1}} e^{i \omega t^{\prime}}\left[\frac{1}{R_{1}}-\frac{i \omega}{c_{n}}\right] \hat{\mathbf{R}}_{1} \cdot \hat{\rho}=-\frac{q}{4 \pi \epsilon} \frac{e^{i k R_{1}}}{R_{1}} e^{i \omega t_{1}} \frac{i}{\omega}\left[\frac{1}{R_{1}}-\frac{i \omega}{c_{n}}\right] \hat{\mathbf{R}}_{1} \cdot \hat{\rho}, \\
-\frac{\partial}{\partial \rho} \Phi_{2}=\frac{q}{4 \pi \epsilon} \frac{e^{i k R_{2}}}{R_{2}} e^{i \omega t_{2}} \frac{i}{\omega}\left[\frac{1}{R_{2}}-\frac{i \omega}{c_{n}}\right] \hat{\mathbf{R}}_{2} \cdot \hat{\rho} .
\end{gathered}
$$

We have dropped the terms with $\delta(\omega)$, once again. Formally, the derivatives with respect to $z$ are the same but making the replacement $\hat{\rho} \rightarrow \hat{z}$. This fact, along with the identity

$$
\hat{\mathbf{R}} \cdot \hat{\rho}+\hat{\mathbf{R}} \cdot \hat{z}=\hat{\mathbf{R}}
$$

and also that $\mu_{0}=1 /\left(\epsilon c_{n}^{2}\right)$, helps us to write the total electric field. Recalling Eqs. (C7), (C10), (C11), and (C12),

$$
\begin{aligned}
\mathbf{E}(\mathbf{x}, \omega)=-\nabla \Phi+i \omega \mathbf{A}= & \frac{q}{4 \pi \epsilon}\left\{-\frac{\hat{\mathbf{R}}_{1}}{R_{1}} e^{i \omega t_{1}} e^{i k R_{1}}\left[\frac{i}{\omega R_{1}}+\frac{1}{c_{n}}\right]+\frac{\hat{\mathbf{R}}_{2}}{R_{2}} e^{i \omega t_{2}} e^{i k R_{2}}\left[\frac{i}{\omega R_{2}}+\frac{1}{c_{n}}\right]\right. \\
& \left.+\int_{t_{1}}^{t_{2}} \mathrm{~d} t^{\prime} \frac{e^{i k R}}{R} e^{i \omega t^{\prime}}\left[\frac{1}{R}-\frac{i \omega}{c_{n}}\right] \hat{\mathbf{R}}+\int_{t_{1}}^{t_{2}} \mathrm{~d} t^{\prime} \frac{e^{i k R}}{R} e^{i \omega t^{\prime}} \frac{i \omega}{c_{n}^{2}} \frac{\mathbf{v}}{R}\right\},
\end{aligned}
$$

which is exactly the same as Eq. (B9). Since we can always find a frame where the track lies along the $z$ axis, and Eq. (C14) is the same as Eq. (B9), that means that Eq. (21) contains the exact field of a particle track at all frequencies. 
[1] S. Buitink et al., Nature (London) 531, 70 (2016).

[2] F. S. Gate, Thesis, Ecole des Mines de Nantes, 2016.

[3] The Pierre Auger Collaboration, Phys. Rev. Lett. 116, 241101 (2016).

[4] F. G. Schröder, Prog. Part. Nucl. Phys. 93, 1 (2017).

[5] G. A. Askaryan, Sov. Phys. JETP 14, 441 (1962).

[6] H. R. Allan, Progress in Elementary Particle and Cosmic Ray Physics (North Holland, Amsterdam, 1971), p. 169.

[7] J. Alvarez-Muñiz, W. Carvalho, Jr., H. Schoorlemmer, and E. Zas, Astropart. Phys. 59, 29 (2014).

[8] A. Bellétoile, R. Dallier, A. Lecacheux, V. Marin, L. Martin, B. Revenu, and D. Torres, Astropart. Phys. 69, 50 (2015).

[9] The Pierre Auger Collaboration, Phys. Rev. D 89, 052002 (2014).

[10] O. Scholten et al., Phys. Rev. D 94, 103010 (2016).

[11] CODALEMA Collaboration, arXiv:1710.02487v1.

[12] The Pierre Auger Collaboration, Phys. Rev. D 93, 122005 (2016).

[13] W. D. Apel et al., Phys. Lett. B 763, 179 (2016).

[14] P. Schellart et al., Astron. Astrophys. 560, A98 (2013).

[15] J. Alvarez-Muñiz, W. R. Carvalho, D. García-Fernández, H. Schoorlemmer, and E. Zas, Astropart. Phys. 66, 31 (2015).

[16] J. Alvarez-Muniz et al., Phys. Rev. D 86, 051104(R) (2012).

[17] H. Schoorlemmer et al., Astropart. Phys. 77, 32 (2016).

[18] J. R. Prescott, J. H. Hough, and J. K. Pidcock, in Proceedings 11th ICRC (Budapest, Hungary, 1970), Vol. 3.

[19] H. R. Allan and R. W. Clay, Nature (London) 225, 253 (1970).

[20] T. J. Stubbs, Nature Phys. Sci. 230, 172 (1971).

[21] J. H. Hough, J. R. Prescott, and R. W. Clay, Nature Phys. Sci. 232, 14 (1971).

[22] H. R. Allan, M. P. Sun, and J. K. Jones, in Proceedings 14th ICRC (Munich, Germany, 1975), p. 3083.

[23] K. Nishi and K. Suga, in Proceedings 20th ICRC (Moscow, Russia, 1987), Vol. 6, p. 125.

[24] K. Kadota, Y. Suzuki, K. Nishi, and F. Kakimoto, in Proceedings 18th ICRC (Calgary, Canada, 1993), Vol. 4, p. 262.

[25] C. Castagnoli et al., in Proceedings 22th ICRC (Dublin, Ireland, 1991), Vol. 4, p. 363.

[26] B. Revenu and V. Marin, in Proceedings 33th ICRC (Rio de Janeiro, Brazil, 2013), p. 398.

[27] R. Baishya et al., NCimC 16, 17 (1993).

[28] V. Marin and B. Revenu, arXiv:1211.3305v1.
[29] A. Escudie et al., in Proceedings 35th ICRC (PoS, Busan, South Korea, 2017), p. 418.

[30] E. Zas, F. Halzen, and T. Stanev, Phys. Rev. D 45, 362 (1992).

[31] R. V. Buniy and J. P. Ralston, Phys. Rev. D 65, 016003 (2001).

[32] V. Marin and B. Revenu, Astropart. Phys. 35, 733 (2012).

[33] J. Alvarez-Muñiz, W. R. Carvalho, and E. Zas, Astropart. Phys. 35, 325 (2012).

[34] T. Huege, M. Ludwig, and C. W. James, AIP Conf. Proc. 1535, 128 (2012).

[35] J. Alvarez-Muñiz, A. Romero-Wolf, and E. Zas, Phys. Rev. D 81, 123009 (2010).

[36] M. E. Peskin and D. V. Schroeder, An Introduction to Quantum Field Theory (Perseus Books Publishing, Reading, MA, 1995).

[37] D. García-Fernández, J. Alvarez-Muñiz, W. R. Carvalho, Jr., A. Romero-Wolf, and E. Zas, Phys. Rev. D 87, 023003 (2013).

[38] G. N. Afanasiev, Vavilov-Cherenkov and Synchrotron Radiation: Foundations and Applications (Kluwer Academic, The Netherlands, 2004).

[39] T. Bergmann, R. Engel, D. Heck, N. Kalmykov, S. Ostapchenko, T. Pierog, T. Thouw, and K. Werner, Astropart. Phys. 26, 420 (2007).

[40] F. Gaté, S. Buitink, J. R. Hörandel, S. de Jong, R. Lahmann, R. Nahnhauer, and O. Scholten, EPJ Web Conf. 135, 01007 (2017).

[41] B. Revenu et al., in Proceedings 35th ICRC (PoS, Busan, South Korea, 2017), p. 1143.

[42] C. W. James, H. Falcke, T. Huege, and M. Ludwig, Phys. Rev. E 84, 056602 (2011).

[43] P. Motloch, J. Alvarez-Muiz, P. Privitera, and E. Zas, Phys. Rev. D 93, 043010 (2016); 94, 049905 (2016).

[44] C. A. Balanis, Antenna Theory, Analysis, and Design, 2nd ed. (John Wiley and Sons, New York, 1997).

[45] P. Abreu et al., J. Instrum. 7, P10011 (2012).

[46] K. A. Michalski and J. R. Mosig, IEEE Trans. Antennas Propag. 63, 5777 (2015).

[47] B. K. Sternberg and T. M. Levitskaya, Radio Sci. 36, 709 (2001).

[48] O. Martineau-Huynh et al., in Proceedings 34th ICRC (PoS, The Hague, Netherlands, 2015), p. 1143. 